\title{
OPEN The composition of Australian Plantago seeds highlights their potential as nutritionally-rich functional food ingredients
}

\begin{abstract}
James M. Cowley ${ }^{1,2}$, Lisa A. O'Donovan² \& Rachel A. Burton ${ }^{1,2}$
When wetted, Plantago seeds become covered with a polysaccharide-rich gel called mucilage that has value as a food additive and bulking dietary fibre. Industrially, the dry husk layer that becomes mucilage, called psyllium, is milled off Plantago ovata seeds, the only commercial-relevant Plantago species, while the residual inner seed tissues are either used for low value animal feed or discarded. We suggest that this practice is potentially wasting a highly nutritious resource and here describe the use of histological, physicochemical, and chromatographic analyses to compare whole seed composition/ characteristics of $P$. ovata with 11 relatives already adapted to harsh Australian conditions that may represent novel commercial crop options. We show that substantial interspecific differences in mucilage yield and macromolecular properties are mainly a consequence of differences in heteroxylan and pectin composition and probably represent wide differences in hydrocolloid functionality that can be exploited in industry. We also show that non-mucilage producing inner seed tissues contain a substantial mannan-rich endosperm, high in fermentable sugars, protein, and fats. Whole seed Plantago flour, particularly from some species obtained from harsh Australian environments, may provide improved economic and health benefits compared to purified $P$. ovata psyllium husk, by retaining the functionality of the seed mucilage and providing additional essential nutrients.
\end{abstract}

Plants of the genus Plantago occur widely throughout the world. Traditionally, all parts of the plant are used ${ }^{1-5}$, but the seeds have particular importance as they produce a gel-like coating of mucilage upon wetting which has many folk food and medicinal uses. For example, seeds of Australian Plantago species were pounded into a flour by Aboriginal and Torres Strait Islander peoples and combined with water to make a porridge thickened by the sticky mucilage ${ }^{6,7}$. Early British settlers also noted the palatability of Australian native Plantago seeds, exploiting the jelly-like mucilage to prepare sweetened desserts similar to sago pudding ${ }^{8}$. More recently, Plantago seed mucilage has gained great industrial and medical significance. Commonly known as psyllium husk, the milled seed mucilage of $P$. ovata contains highly-hydrophilic heteroxylan and pectin polysaccharides ${ }^{9,10}$ that are used in food technology to texturally mimic fat ${ }^{11}$ and gluten ${ }^{12,13}$, and in health applications as a dietary fibre supplement to aid laxation ${ }^{14}$, treat hypercholesterolemia ${ }^{15}$, diabetes ${ }^{16}$, and irritable bowel syndrome ${ }^{17}$. While the clinical benefits of psyllium husk (generally attributed to the high viscosity of the heteroxylan) are not in doubt, pre-clinical and in vitro studies showing free radical scavenging ${ }^{18-23}$, immunomodulation ${ }^{24-26}$, and treatment of metabolic disorders ${ }^{27-30}$ by extracts of whole, unhusked Plantago seeds demonstrate that the non-husk/nonmucilage seed components may have further beneficial effects. However, current production of psyllium husk from $P$. ovata (the only commercially important Plantago species) mostly discards these non-husk/non-mucilage components which account for around $75 \%$ of the seed yield ${ }^{10}$. In India (the world's largest psyllium producer) this equates to over 160,000 tonnes of non-husk material that was wasted from the 56,000 tonnes of psyllium husk produced in $2019 / 2020^{31,32}$ despite the material likely containing many beneficial nutrients. It is likely that a major factor contributing to the underutilisation of whole Plantago seeds is that remarkably little is known about whole seed composition, particularly from a nutritional perspective, and how the composition differs between species. Natural interspecific variation in the cosmopolitan Plantago genus has already been a valuable resource to study polysaccharide biosynthesis ${ }^{33-35}$ but is now increasing in interest as a source of novel ingredients with

\footnotetext{
${ }^{1}$ Australian Research Council Centre of Excellence in Plant Cell Walls, School of Agriculture, Food and Wine, University of Adelaide, Waite Campus, Urrbrae, SA, Australia. ${ }^{2}$ Australian Research Council Centre of Excellence in Plant Energy Biology, School of Agriculture, Food and Wine, University of Adelaide, Waite Campus, Urrbrae, SA, Australia. ${ }^{\varpi}$ email: rachel.burton@adelaide.edu.au
} 
unique functional properties for industry ${ }^{19,36-41}$. The limited studies that are currently available have shown that whole seed flour (WSF) from $P$. ovata and $P$. psyllium are useful hydrocolloid replacements in baking ${ }^{42,43}$ with significant differences in product quality between the two species. These studies show the potential of WSF from different Plantago species to be a less wasteful, more nutritious alternative to psyllium husk for selectively manipulating food qualities based on interspecific differences in functionality. Therefore, we hypothesise that different Plantago species may yield correspondingly diverse functionalities and Australia may boast valuable untapped resources, with the added advantage of already being adapted to demanding climatic constraints that limit production of psyllium from $P$. ovata ${ }^{10}$.

Thus, the aim of this work was to compare the composition and morphology of seeds of commercial psyllium, $P$. ovata, to eleven related species naturalised or native to Australia to assess their nutritional and functional potential as novel food ingredients. The results demonstrate great diversity in mucilage properties and composition that likely represent exploitable differences in hydrocolloid functionality and have provided the first comprehensive overview of Plantago seed composition, showing them to be rich in beneficial sugars, fats and protein. We suggest that the use of WSF from diverse Plantago species studied here will simultaneously allow selective manipulation of quality and nutritional enrichment of food products.

\section{Materials and methods}

Plant growth for seed bulking. Seeds of twelve Plantago species were obtained from the sources listed in Supplementary Table 1, and where applicable, collection complied with relevant institutional, national, and international guidelines and legislation. Seeds were stratified dry for $48 \mathrm{~h}$ at $-20{ }^{\circ} \mathrm{C}$ prior to germination then imbibed in filtered $(0.22 \mu \mathrm{m})$ sterilisation agent $(50: 50,50 \%$ ethanol:4\% bleach with $0.05 \%$ Triton X-100) for $1 \mathrm{~min}$ before replacing the sterilisation agent and incubating for another minute. This was repeated until the seeds had been imbibed in fresh sterilisation agent 5 times, after which the seeds were washed 5 times with filtersterilised $(0.22 \mu \mathrm{m})$ Milli-Q water. Seeds were spread onto pre-wetted autoclaved Whatman No. 1 paper in a sterile petri dish. Dishes were sealed, aluminium foil-wrapped and stratified for another $48 \mathrm{~h}$ at $4^{\circ} \mathrm{C}$. After stratification, plates were moved to a glasshouse with a day/night temperature of $23^{\circ} \mathrm{C} / 18^{\circ} \mathrm{C}$. Seeds were germinated for 10 days ( 3 days dark then 7 days exposed to the glasshouse day/night light cycle) then transferred to coco-peat soil mixture in tall citrus pots. Plants were grown to maturity from June to December (Adelaide, Australia) with no supplemental light. Once mature (capsules freely dehiscing), inflorescences were harvested, dried for $48 \mathrm{~h}$ at $37^{\circ} \mathrm{C}$, and then threshed to obtain seed. Where required, whole seeds were ground using a MM400 Mixer Mill (Retsch, Germany) and graded to $0.5 \mathrm{~mm}$ to produce whole seed flour (WSF).

Phylogenetic analysis. Mature leaf tissue from Plantago plants was frozen at $-80^{\circ} \mathrm{C}$ and ground by stainless steel ball bearing for $30 \mathrm{~s}$ at $30 \mathrm{~Hz}$ using a MM400 Mixer Mill (Retsch, Germany) fitted with $2 \mathrm{ml}$ tube adapter. DNA was extracted from ground leaf tissue following Healey et al. ${ }^{44}$. Nuclear ribosomal internal transcribed spacer (ITS) regions were amplified by PCR using primers ${ }^{45}$ and conditions listed in Supplementary Table 2. Amplified ITS regions were sequenced by AGRF (Adelaide, Australia). Where possible, sequence identities were confirmed by MUSCLE nucleotide alignment with published sequences from Rønsted et al. ${ }^{46}$ and Tay et al. ${ }^{47}$. The ITS sequence of Veronica salicornioides, FJ024624. $1^{47}$, was downloaded from Genbank and used as an outgroup as per Rønsted et al. ${ }^{46}$.

ITS sequences were trimmed using $\mathrm{BMGE}^{48}$ and the maximum-likelihood tree constructed in Geneious v8.1.3 (Biomatters Ltd, NZ) with the RAxML tree builder ${ }^{49}$ using the GTR GAMMA nucleotide model with 500 rapid bootstrapping replicates.

Seed morphometric measurements. Seed length and width measurements were determined by image analysis. Images of seeds were taken at $1 \times$ magnification on an AxioImager M2 (Zeiss, Germany) fitted with an AxioCam 105 colour camera (Zeiss, Germany). Length and width of 20 seeds per species were measured using ZEN 2012 software (Zeiss, Germany). To determine 1000 seed weight, seeds were manually counted and weighed.

Mucilage microscopy. Mucilage architecture was observed by whole mount immunolocalisation of heteroxylan using the LM11 antibody ${ }^{50}$ and ruthenium red staining following Phan et al. ${ }^{33}$. Ruthenium red used in this work (C075) was purchased from ProSciTech (Australia) and prepared as per Cowley et al. ${ }^{51}$.

Whole seed thin section microscopy and immunolabelling. Whole seeds were transversely halved with a razor blade and fixed and embedded in LR White resin as per Burton et al..$^{52}$ modified to use the $80 \%$ ethanol fixative described in Phan et al. ${ }^{9}$. Fixed and embedded tissue was sectioned at $1 \mu \mathrm{m}$ on an Ultramicrotome (Leica, EM UC6) using a diamond knife (DiATOME, Nidau, Switzerland). After staining thin sections with toluidine blue, internal seed structures were imaged with an AxioImager M2 (Zeiss, Germany) fitted with an AxioCam 105 colour camera (Zeiss, Germany). Endosperm mannan polysaccharides were immunolocalised using a tenfold dilution of LM21 anti- $(1,4)-\beta$-mannan primary antibody (Kerafast, US). The secondary antibody, Alexa Fluor 555 goat anti-rat IgG, was applied at a 100-fold dilution. All fluorescent images were taken with a Zeiss M2 AxioImager with an AxioCam 506 mono black and white camera. Images were processed using ZEN 2012 software (Zeiss, Germany).

Seed mucilage fractionation and yield analysis. Mucilage was extracted and fractionated and yield traits were determined following Cowley et al. ${ }^{51}$ with no deviation from the described procedure. Briefly, $1 \mathrm{~mL}$ 
of water was added to $30 \mathrm{mg}$ of seed and extracted at $25^{\circ} \mathrm{C}$ for $1.5 \mathrm{~h}$ with agitation. After brief centrifugation, the supernatant was transferred to a new tube (cold water extractable (CWE) mucilage fraction) and the volume of the pellet-containing tube was returned to $1 \mathrm{~mL}$. The extraction was repeated again, but at $65^{\circ} \mathrm{C}$ to obtain the hot water extractable (HWE) mucilage fraction. Finally, the pellet (seeds with the most extraction resistant mucilage fraction) were agitated on a tissue disruptor-type mill at $30 \mathrm{~Hz}$ for $10 \mathrm{~min}$ to obtain the intense agitation extractable (IAE) fraction. Collected fractions were freeze-dried to a constant weight and compared to starting mass of seeds to determine the yield:

$$
\text { Yield }(\%)=\left(\frac{\text { mass of freeze dried mucilage }}{\text { mass of seeds pre-extraction }}\right) \times 100
$$

Water absorption capacity assay. The water absorption capacity was determined following Cowley et al. ${ }^{51}$ with modifications. As the seeds were of variable size, $30 \mathrm{mg}$ were weighed into $2 \mathrm{~mL}$ microcentrifuge tubes and $1 \mathrm{~g}$ of deionized water was added to each tube. After briefly vortexing to break surface tension and submerge seeds, the seed mucilage was allowed to expand, undisturbed, for $45 \mathrm{~min}$ at room temperature $\left(25^{\circ} \mathrm{C}\right)$. After $45 \mathrm{~min}$, a $1000 \mu \mathrm{L}$ laboratory pipette was used to remove unabsorbed water, avoiding disturbing the seeds and their mucilage. Removed water was weighed and the water absorption capacity was determined using the following equation:

$$
\text { Water absorption capacity }(\mathrm{g} / \mathrm{g})=\frac{\text { Initial weight of water added }- \text { weight of unabsorbed water }}{\text { Initial weight of seeds added }}
$$

Monosaccharide analysis. Monosaccharide profiles of fractionated mucilage (redispersed at $1 \mathrm{mg} / \mathrm{mL}$ in Milli-Q water) and WSF were determined using reverse phase high performance liquid chromatography (RPHPLC) of 1-phenyl-3-methyl-5-pyrazoline (PMP) derivatives following Cowley et al. ${ }^{51}$. Area under the peaks was compared to standard curves of mannose, ribose, rhamnose, glucuronic acid, galacturonic acid, glucose, galactose, xylose, arabinose and fucose ${ }^{53}$.

Whole seed soluble sugar extraction and profiling. Soluble sugars were extracted from $10 \mathrm{mg}$ of WSF following Vespreet et al. ${ }^{54}$ with modifications based on Matros et al. ${ }^{55}$. Due to substantial mucilage gelling in water when attempting a sequential $80 \%$ ethanol and water extraction, soluble sugars were twice extracted from flour in $80 \%$ ethanol at $85^{\circ} \mathrm{C}$ for $30 \mathrm{~min}$ on a mixer $(700 \mathrm{rpm})$ at a final dilution of $1: 40(\mathrm{w} / \mathrm{v}, \mathrm{mg} / \mu \mathrm{l})$. Supernatants were combined, diluted with water to $1: 25,000(\mathrm{w} / \mathrm{v}, \mathrm{mg} / \mu \mathrm{l})$ and $25 \mu \mathrm{l}$ per sample were analysed by high $\mathrm{pH}$ anion exchange chromatography with pulsed amperometric detection (HPAEC-PAD) on a Dionex ICS-5000 system using a DionexCarboPAC ${ }^{\mathrm{TM}} \mathrm{PA}-20$ column $(3 \times 150 \mathrm{~mm})$ with a guard column $(3 \times 50 \mathrm{~mm})$ kept at $30^{\circ} \mathrm{C}$ and operated at a flow rate of $0.5 \mathrm{~mL} \mathrm{~min} \mathrm{~m}^{-1}$. The eluents used were (A) $0.1 \mathrm{M}$ sodium hydroxide and (B) $0.1 \mathrm{M}$ sodium hydroxide with $1 \mathrm{M}$ sodium acetate. The gradient used was: $0 \%$ (B) from 0 to $2 \mathrm{~min}, 20 \%$ (B) from 2 to $35 \mathrm{~min}, 100 \%$ (B) from 35 to $36.5 \mathrm{~min}, 0 \%$ (B) from 37.5-38.5 min. Detector temperature was maintained at $20^{\circ} \mathrm{C}$, data collection was at $2 \mathrm{~Hz}$ and the Gold Standard PAD waveform (std. quad. potential) was used.

Data acquisition, processing, and peak integration were performed using the Chromeleon ${ }^{\text {Tx }}$ version 7.1.3.2425 software (Thermo Scientific). Compounds were annotated and quantified based on peaks of available analytical standards. Glucose, fructose, sucrose, raffinose, 1-kestose, maltose, nystose and stachyose analytical standards were purchased from Sigma-Aldrich, while 1,1,1-kestopentaose was obtained from Bio-Strategy. A standard of planteose was purified and prepared from Salvia hispanica seed mucilage as per Xing et al. ${ }^{56}$. Schematic structures were prepared using the DrawGlycan-SNFG online tool ( http://www.virtualglycome.org/DrawGlycan/) ${ }^{57}$.

Protein analysis. Protein content of $150 \mathrm{mg}$ of WSF was determined by the Dumas method using a Rapid $\mathrm{N}$ Exceed bench top nitrogen analyser (Elementar, USA). Conversion factor was 6.25. Protein content was determined in triplicate.

Lipid analysis. Total lipid in $500 \mathrm{mg}$ of WSF was determined by modified Folch method ${ }^{58}$ and fatty acid profiles were determined by gas chromatography of transesterified lipids following Liu et al. ${ }^{59}$. Analyses were performed in duplicate at SAHMRI (Adelaide, Australia). Multivariate principle component analysis for separation was performed in PAST software (version 3.25) ${ }^{60}$.

Energy calculation. Energy content of whole seeds was estimated from the energy density of protein, carbohydrate, dietary fibre and fat components in whole Plantago seed ${ }^{61}$. Average protein, carbohydrate, dietary fibre, and fat contents $(\% \mathrm{w} / \mathrm{w})$ were obtained from whole seed protein, soluble sugar, monosaccharide, and lipid profiling analyses, respectively.

Figures. Unless otherwise indicated, plots were generated using Prism 8.4 (GraphPad, USA).

Consent for publication. All authors give consent for the data to be published. 
A
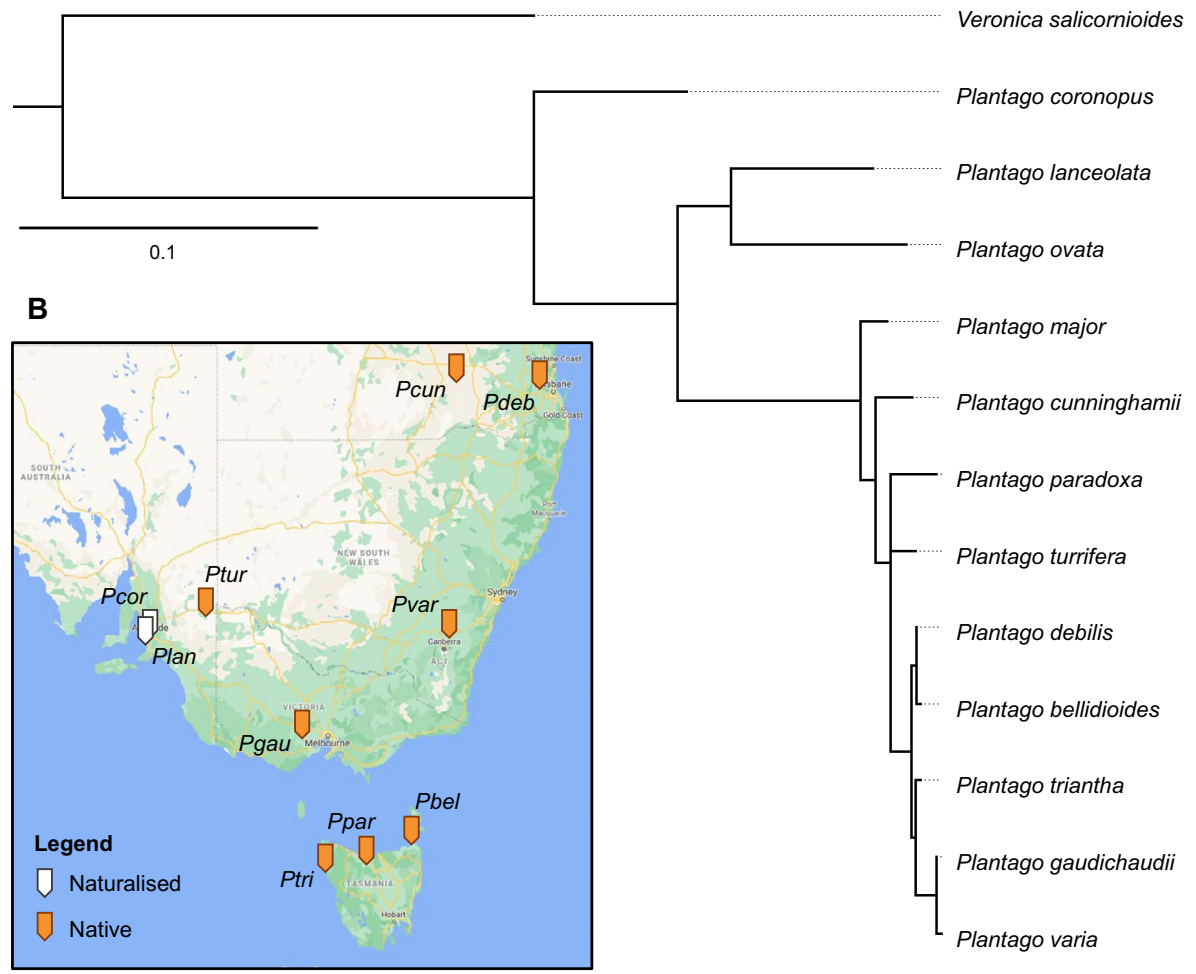

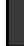
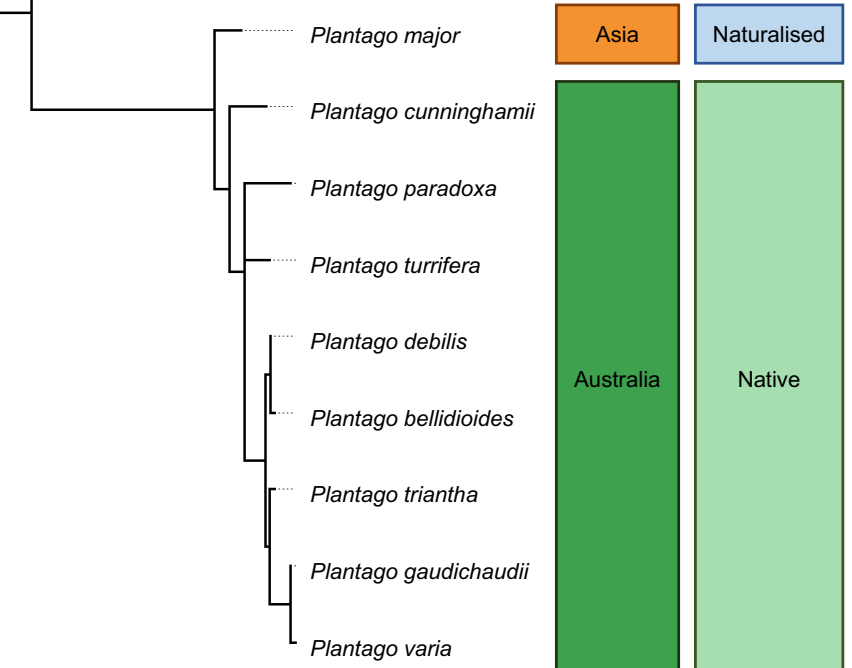

Figure 1. (A) Maximum-likelihood tree of nuclear ribosomal DNA internally transcribed spacer (ITS) regions of Plantago species studied here. The ITS sequence of Veronica salicornioides, a New Zealand (NZ)-endemic relative in Plantaginaceae, is included as the outgroup. Noted alongside each species are their geographic origin (where native to) and the status of occurrences in Australia (naturalised, native or grown commercially). Scale refers to substitutions per site. (B) Locations of Plantago sources in South Eastern Australia based on coordinates supplied with seedbank-withdrawn accessions or collection sites. Map sourced from Google Earth earth.google.com/web. P. ovata and P. major samples were purchased commercially, so origin information can only be assumed. Abbreviations: Pbel $=P$. bellidioides; $P$ cor $=P$. coronopus; $P c u n=P$. cunninghamii; $P d e b=P$. debilis; Pgau = P. gaudichaudii; Plan = P. lanceolata Ppar $=$ P. paradoxa Ptri $=$ P. triantha Ptur $=$ P. turrifera; Pvar $=$ P. varia.

\section{Results}

Genetic relationships. A maximum-likelihood tree of nuclear ribosomal internally transcribed spacer (ITS) region sequences ${ }^{46,47}$ of the Plantago species studied here was used to produce a phylogenetic tree (Fig. 1). The species can be separated into clades by their origin, $P$. coronopus from Europe, P. lanceolata and P. ovata from Central Europe and Eurasia, and the remaining species from Australasia. The Australasian Plantago species, particularly Australian natives, are more genetically-similar than the other species studied here, but P. major, a naturalised species, is grouped into an Asian clade.

Using the tree in Fig. 1, the 12 species were ranked based on their relative genetic distance from the outgroup and each other and this order is used in all subsequent figures to allow observation of trends between more distantly and more closely-related species.

Seed mucilage characteristics. There is significant variation in expanded seed mucilage architecture of the Plantago species studied here (Fig. 2). We corroborate the same characteristics and differences in ruthenium red phenotype of $P$. coronopus, $P$. lanceolata, $P$. ovata, $P$. major, $P$. cunninghamii, $P$. debilis and $P$. varia described in detail by Phan et al. ${ }^{33}$. Here we studied five additional Australian native species and found, owing to their relatedness (Fig. 1), P. turrifera and P. bellidioides to be strikingly similar to $P$. cunninghamii and $P$. debilis, and $P$. gaudichaudii to be most similar to $P$. varia. These similarities were also found when immunolabelling the mucilage (Fig. 2a-1). Two further native species, $P$. paradoxa and $P$. triantha, despite their apparent genetic similarity to other native species (Fig. 1), produced a negligible mucilage envelope that was poorly distinguishable with ruthenium red staining (Fig. 2F and 3J) and only slightly more apparent with xylan-directed immunolabelling (Fig. $2 \mathrm{f}$ and $3 \mathrm{j}$ ).

In this study we used our previously published mucilage fractionation method ${ }^{51}$ to investigate mucilage yield and fractionation traits (Fig. 3). There was significant interspecific variation in total yield (Fig. 3A) and fractionation profile (Fig. 3B) of seed mucilage. Total yield of mucilage was highest in P. ovata and comparatively lower in P. lanceolata, $P$. paradoxa, and P. triantha. Australian native species, $P$. cunninghamii, $P$. turrifera, $P$. bellidioides, P. debilis, P. gaudichaudii and P. varia had quite similar total yield (Fig. 3A) and fractionation profiles (Fig. 3B) 


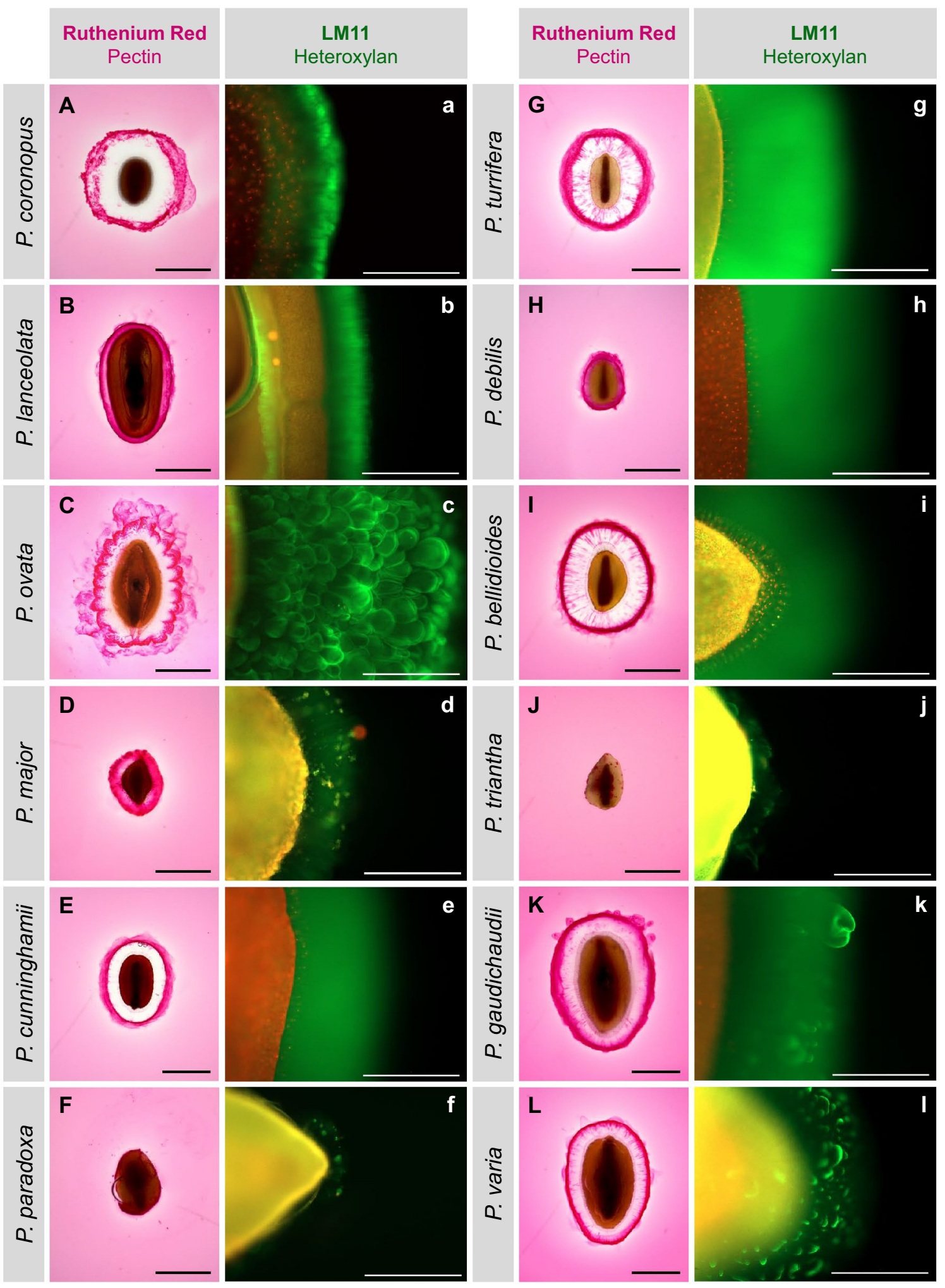

Figure 2. Expanded mucilage architecture of 12 Plantago species. Ruthenium red (dark pink) stains acidic polysaccharides at the mucilage periphery $(\mathbf{A}-\mathbf{L})$. Scale $=1 \mathrm{~mm}$. Anti-xylan antibody LM11 (green) probes for xylan backbone epitopes in the inner mucilage layers (a-l) while the seed is counterstained with propidium iodide (red/yellow). Scale $=500 \mu \mathrm{m}$. 
A
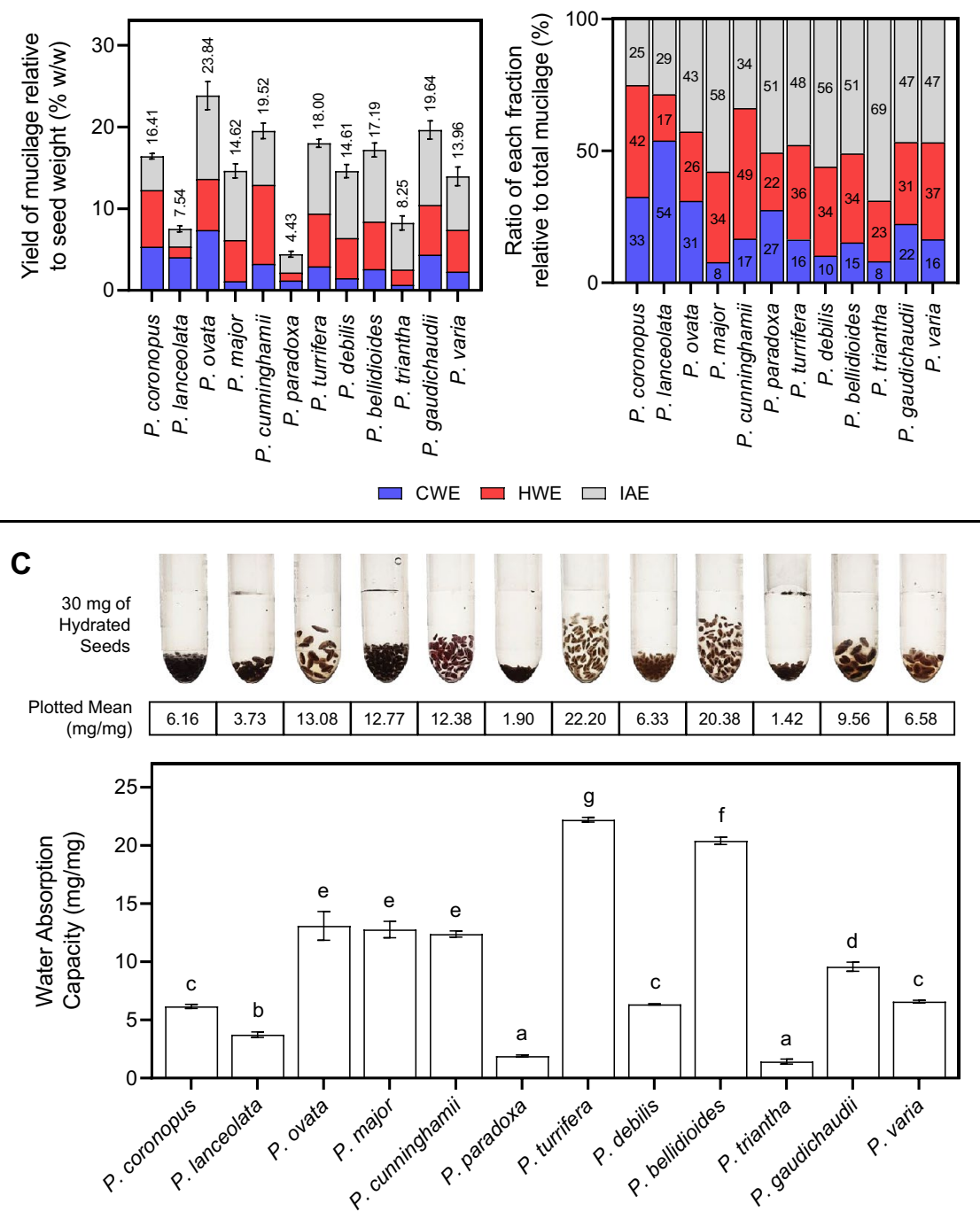

D
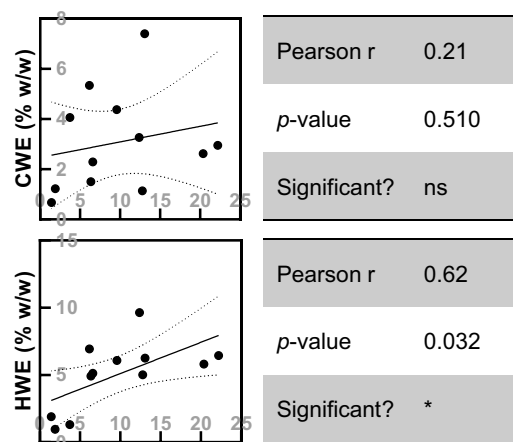

$p$-value

0.510

Significant? ns

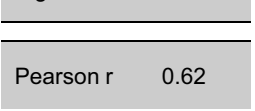

$p$-value $\quad 0.032$

Significant? *
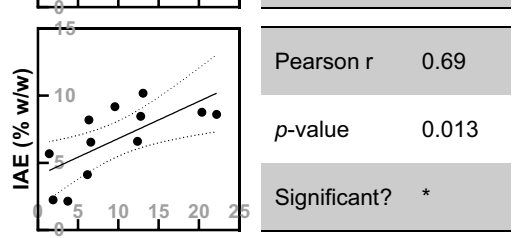

$p$-value

0.013

Significant? *
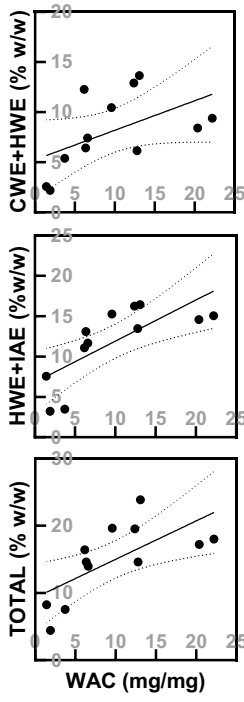

Significant? ns

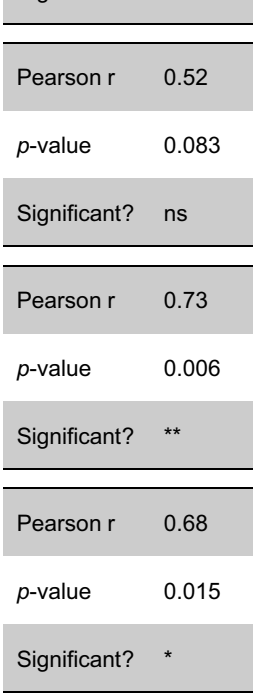

Figure 3. Seed mucilage yield, fractionation and water absorption traits of 12 Plantago species. (A) Yield of mucilage as a fraction of the whole seed. (B) Quantity of each isolated mucilage fraction as share of total mucilage. Values within bars are plotted values rounded to the nearest integer. $\mathrm{CWE}=$ cold water extractable (blue); HWE = hot water extractable (red); IAE = intense agitation extractable (grey). (C) Water absorption capacity of Plantago seeds. Above the plot are plotted mean values and a representative image of $30 \mathrm{mg}$ of hydrated and swollen Plantago seeds. Samples sharing a letter are not significantly different from each other $(p>0.05)$. (D) Study of correlations between water absorption capacity (WAC) and mucilage yield/fractionation traits (seed-relative traits from A). Linear models plotted with $95 \%$ confidence bands. ns = not significant. Values presented are means of five replicates in $(\mathbf{A})$ and $(\mathbf{B})$ and three replicates in $(\mathbf{C})$ Error bars correspond to one standard deviation.

and less mucilage could be extracted using cold water (CWE). To complement mucilage extractability traits, the water absorption capacity by whole Plantago seeds was determined (Fig. 3C). Water absorption capacity (WAC) was found to vary substantially with $P$. turrifera and $P$. bellidioides having a significantly higher WAC than other species, while absorption by $P$. lanceolata, $P$. paradoxa and $P$. triantha was very low. A correlation study was performed to determine if there was a link between mucilage fractionation and yield traits and the observed differences in WAC (Fig. 3D). Logically, significant $(p<0.05)$ correlations between WAC and total mucilage content and WAC and the more gel-like extraction resistant mucilage fractions were found. The strongest correlation $(\mathrm{r}=0.73, p=0.006)$ with WAC was the proportion of hot water- and intense agitation extractable (HWE + IAE) mucilage.

The chemical composition of fractionated mucilage was determined by monosaccharide profiling which revealed both interspecific differences and trends (Fig. 4). Monosaccharide profiles were similar between all fractions of all species, with xylose and arabinose (constituents of heteroxylan) comprising the majority of monosaccharides quantified along with rhamnose and galacturonic acid (constituents of pectin) and minor quantities of glucose and galactose (Fig. 4A). Other monosaccharides for which standards were included, mannose, 


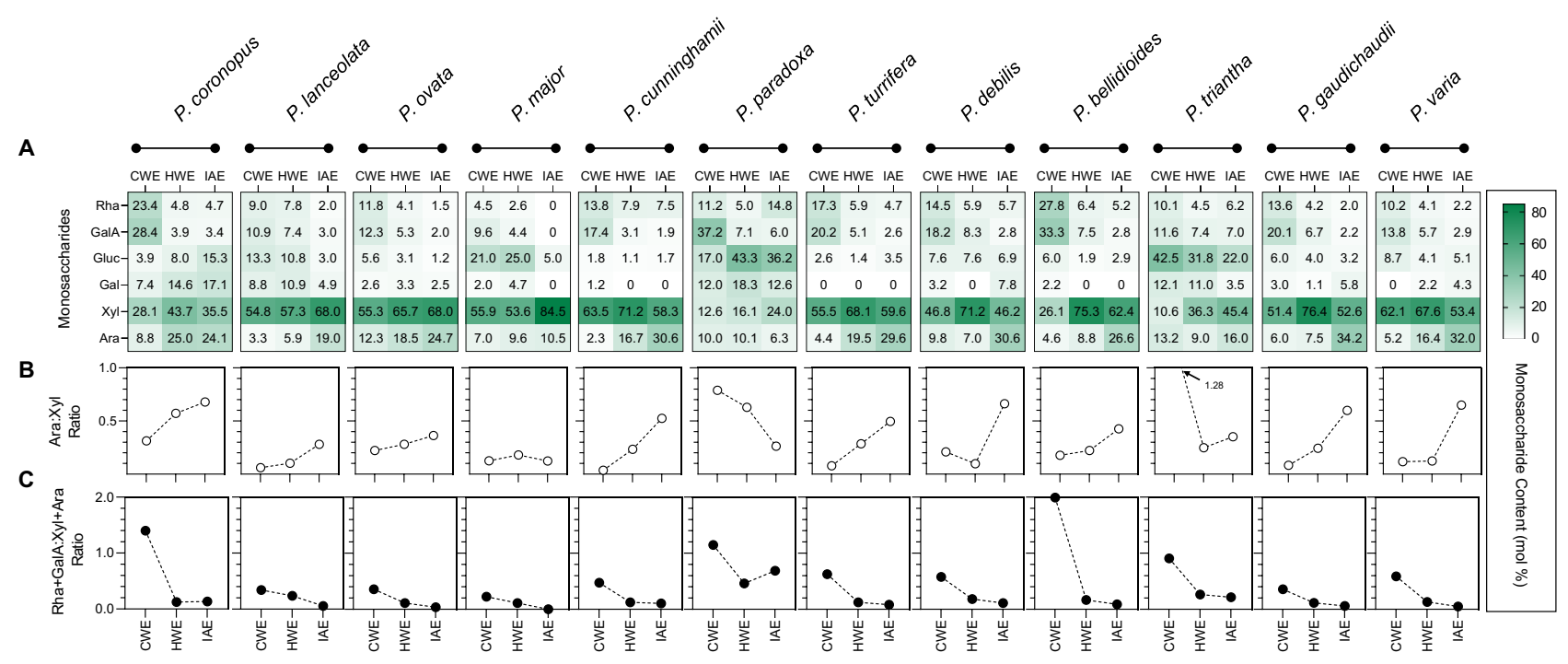

Figure 4. Chemical composition of fractionated mucilage from 12 Plantago species. (A) Monosaccharide composition of each mucilage fraction. Heatmap of relative (molar) abundance of each monosaccharide in a fraction. (B) Arabinose to xylose (Ara:Xyl) ratio in each fraction. (C) Pectin (rhamnose and galacturonic acid) to heteroxylan (xylose and arabinose) $(\mathrm{Rha}+\mathrm{GalA}: \mathrm{Xyl}+\mathrm{Ara})$ ratio in each fraction. $\mathrm{CWE}=$ cold water extractable; HWE = hot water extractable; IAE = intense agitation extractable. Values presented are the means of five fractionation preps.

ribose, glucuronic acid, and fucose, were minute or not detected (data not shown). The relative abundance of each monosaccharide detected in the mucilage fractions, however, differed significantly. The arabinose to xylose ratio differed interspecifically and also between fractions. In almost all species, the arabinose to xylose ratio rises with increasingly harsh extraction steps (Fig. 4B). In P. paradoxa, the inverse was observed and in the CWE fraction of $P$. triantha the value was outside the expected range of less than 1 (1.28). These species produced little mucilage and displayed poor monosaccharide recovery which explains some of the discrepancies. In particular, the CWE mucilage fraction from $P$. triantha was very small and the detected arabinose and xylose are likely not heteroxylan-derived. Values for the more abundant HWE and IAE fractions are within the expected range $(<1)$. The ratio of rhamnose to galacturonic acid was stable between fractions and differed minimally between species (Fig. 4A). Rhamnose and galacturonic acid, however, were found to be highly-enriched in the CWE fraction of all species (Fig. 4C) but much lower or even absent in subsequent fractions.

Plantago endosperm and embryo morphology and composition. Monosaccharide profiling revealed that mannose is a major non-cellulosic monosaccharide in whole seeds of all species, being the most abundant in 8 of 12 species studied here (Fig. 5A). Using a monoclonal antibody recognising $\beta$ - $(1,4)$-mannan ${ }^{62}$ it was observed that Plantago species have a substantial endosperm with thick (grand median $=8.206 \mu \mathrm{m}$, Supplementary Fig. 1) mannan-rich walls (Fig. 5C-L). Minimal amounts of mannan were detected in the embryo but it was completely absent in the mucilage layer of all species except P. cunninghamii (Fig. 5G). However, mannose is completely absent in P. cunninghamii mucilage, even that tightly adhered to the seed ${ }^{51}$ suggesting that the mannan labelling seen in the mucilage layer of $P$. cunninghamii is likely an artefact of non-specific binding in poorly-fixed mucilage. The endosperm cells also all contained tightly-packed spherical bodies (Supplementary Fig. 2) which are likely to be aleurone grains and/or oil bodies, similar to those seen in seed tissues of other species like Cannabis sativa ${ }^{63}$.

Soluble sugar profiling. Soluble sugar profiling was used to determine the content of rapidly-mobile non-starch reserve carbohydrates in the internal Plantago seed tissues (Fig. 6). Heat mapped chromatographic data (Fig. 6A) show that the soluble sugar profiles are similar between all species with common major components, with most of the interspecific variation coming from differing abundances of the major sugars, sucrose $(\beta$-D-fructofuranosyl- $(2 \rightarrow 1)$ - $\alpha$-D-glucopyranoside) and planteose $(\alpha$-D-galactopyranosyl- $(1 \rightarrow 6)-\beta$-Dfructofuranosyl- $(2 \rightarrow 1)$ - $\alpha$-D-glucopyranoside) which account for up to $86 \%$ of the soluble sugar content (Supplementary Fig. 3). Some variation between profiles was evident between 2 and 5 min elution times but these components are (based on relative peak area; Supplementary Fig. 3) lowly abundant minor sugars, possibly unidentified mono- or di-saccharides. Quantification of extracted soluble sugars shows that the trisaccharide planteose is the major soluble sugar in seeds of the Plantago species studied here (up to $3.2 \% \mathrm{w} / \mathrm{w}$ ), except in P. lanceolata where sucrose dominates, although followed by planteose (Fig. 6B). P. lanceolata also had the most unique soluble sugar profile in other ways: the trisaccharide raffinose $(\beta$-D-fructofuranosyl- $(2 \rightarrow 1)-\alpha$-Dglucopyranoside- $(1 \rightarrow 6)$ - $\alpha$-D-galactopyranoside) and its higher homologue, the tetrasaccharide stachyose $(\beta$-Dfructofuranosyl- $(2 \rightarrow 1)$ - $\alpha$-D-glucopyranoside- $(1 \rightarrow 6)$ - $\alpha$-D-galactopyranosyl- $(1 \rightarrow 6)$ - $\alpha$-D-galactopyranoside), which, present in minute quantities in other species, were additional major soluble sugars in P. lanceolata. 
A

\begin{tabular}{rcccccccc}
\hline & Mannose & Rhamnose & $\begin{array}{c}\text { Glucuronic } \\
\text { Acid }\end{array}$ & $\begin{array}{c}\text { Galacturonic } \\
\text { Acid }\end{array}$ & Glucose & Galactose & xylose & Arabinose \\
\hline P. coronopus & $11.43 \pm 1.39$ & $1.71 \pm 0.25$ & nd & $1.23 \pm 0.18$ & $3.05 \pm 0.45$ & $3.07 \pm 0.32$ & $5.52 \pm 0.72$ & $5.39 \pm 0.68$ \\
P. lanceolata & $19.39 \pm 0.12$ & $0.62 \pm 0.01$ & nd & $0.65 \pm 0.00$ & $5.23 \pm 0.11$ & $2.56 \pm 0.04$ & $3.80 \pm 0.05$ & $3.22 \pm 0.04$ \\
P. ovata & $12.67 \pm 0.99$ & $1.22 \pm 0.02$ & nd & $1.08 \pm 0.02$ & $2.35 \pm 0.12$ & $3.40 \pm 0.10$ & $18.83 \pm 0.49$ & $11.86 \pm 0.08$ \\
P. major & $11.84 \pm 0.97$ & $0.70 \pm 0.04$ & $0.65 \pm 0.03$ & $0.56 \pm 0.01$ & $5.98 \pm 0.29$ & $2.17 \pm 0.05$ & $8.85 \pm 0.05$ & $3.44 \pm 0.08$ \\
P. cunninghamii & $15.35 \pm 0.58$ & $3.19 \pm 0.33$ & nd & $1.15 \pm 0.30$ & $2.83 \pm 0.20$ & $1.50 \pm 0.02$ & $16.15 \pm 1.36$ & $8.32 \pm 0.57$ \\
P. paradoxa & $12.76 \pm 0.13$ & $1.58 \pm 0.05$ & nd & $0.50 \pm 0.00$ & $4.45 \pm 0.14$ & $2.53 \pm 0.07$ & $1.16 \pm 0.01$ & $2.20 \pm 0.02$ \\
P. turrifera & $18.67 \pm 0.88$ & $1.88 \pm 0.08$ & nd & $1.19 \pm 0.08$ & $3.01 \pm 0.19$ & $1.92 \pm 0.11$ & $14.05 \pm 1.11$ & $7.64 \pm 0.66$ \\
P. debilis & $18.18 \pm 0.05$ & $2.09 \pm 0.03$ & nd & $0.76 \pm 0.00$ & $3.97 \pm 0.01$ & $3.06 \pm 0.02$ & $8.23 \pm 0.21$ & $6.11 \pm 0.17$ \\
P. bellidioides & $12.02 \pm 0.76$ & $2.45 \pm 0.05$ & nd & $1.49 \pm 0.03$ & $2.74 \pm 0.01$ & $2.22 \pm 0.02$ & $13.02 \pm 0.39$ & $6.87 \pm 0.14$ \\
P. triantha & $20.25 \pm 0.55$ & $1.41 \pm 0.03$ & nd & $0.81 \pm 0.02$ & $4.77 \pm 0.19$ & $2.16 \pm 0.06$ & $3.14 \pm 0.12$ & $2.83 \pm 0.14$ \\
P. gaudichaudii & $11.91 \pm 0.14$ & $1.46 \pm 0.01$ & nd & $1.18 \pm 0.02$ & $3.98 \pm 0.04$ & $2.74 \pm 0.01$ & $13.59 \pm 0.05$ & $7.94 \pm 0.05$ \\
P. varia & $11.16 \pm 0.20$ & $1.55 \pm 0.01$ & nd & $0.99 \pm 0.01$ & $3.67 \pm 0.00$ & $3.12 \pm 0.01$ & $9.93 \pm 0.14$ & $6.93 \pm 0.07$ \\
\hline
\end{tabular}

B
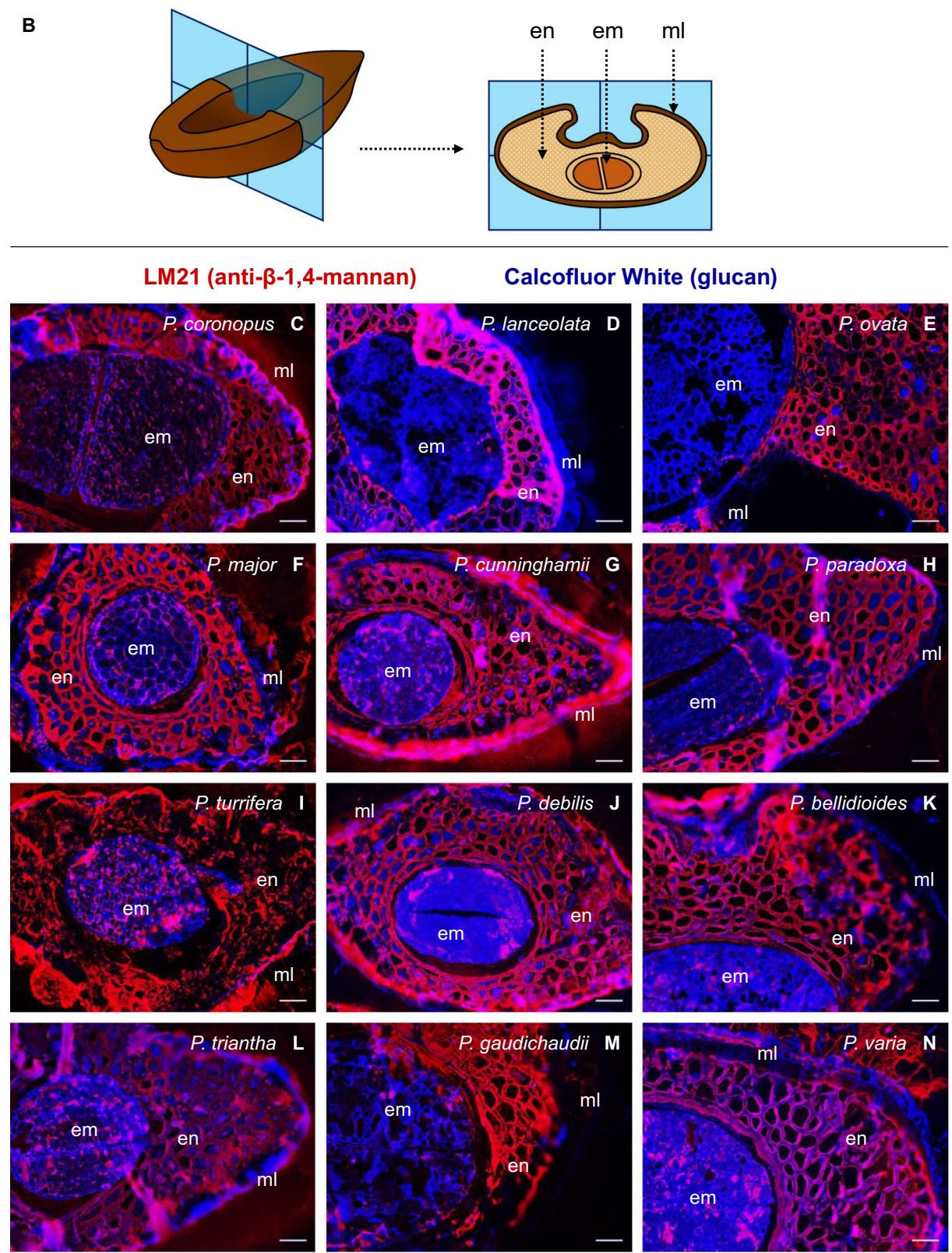

Figure 5. Profiling of the major polysaccharide sugars in Plantago seed. (A) Monosaccharide profiles of whole Plantago seeds (means of three replicates, \% w/w, with standard deviation). A grey scale (dark grey, highest value; white, lowest value) has been used to highlight the most abundant monosaccharides in a species. (B) Schematic diagram of the orientation of thin sections taken from Plantago seeds. (C-N) Immunodetection of mannan in Plantago endosperm cell walls by LM21 monoclonal antibody. Scale $=50 \mu \mathrm{m}$. Abbreviations: en = endosperm; em = embryo; $\mathrm{ml}=$ mucilage layer. 


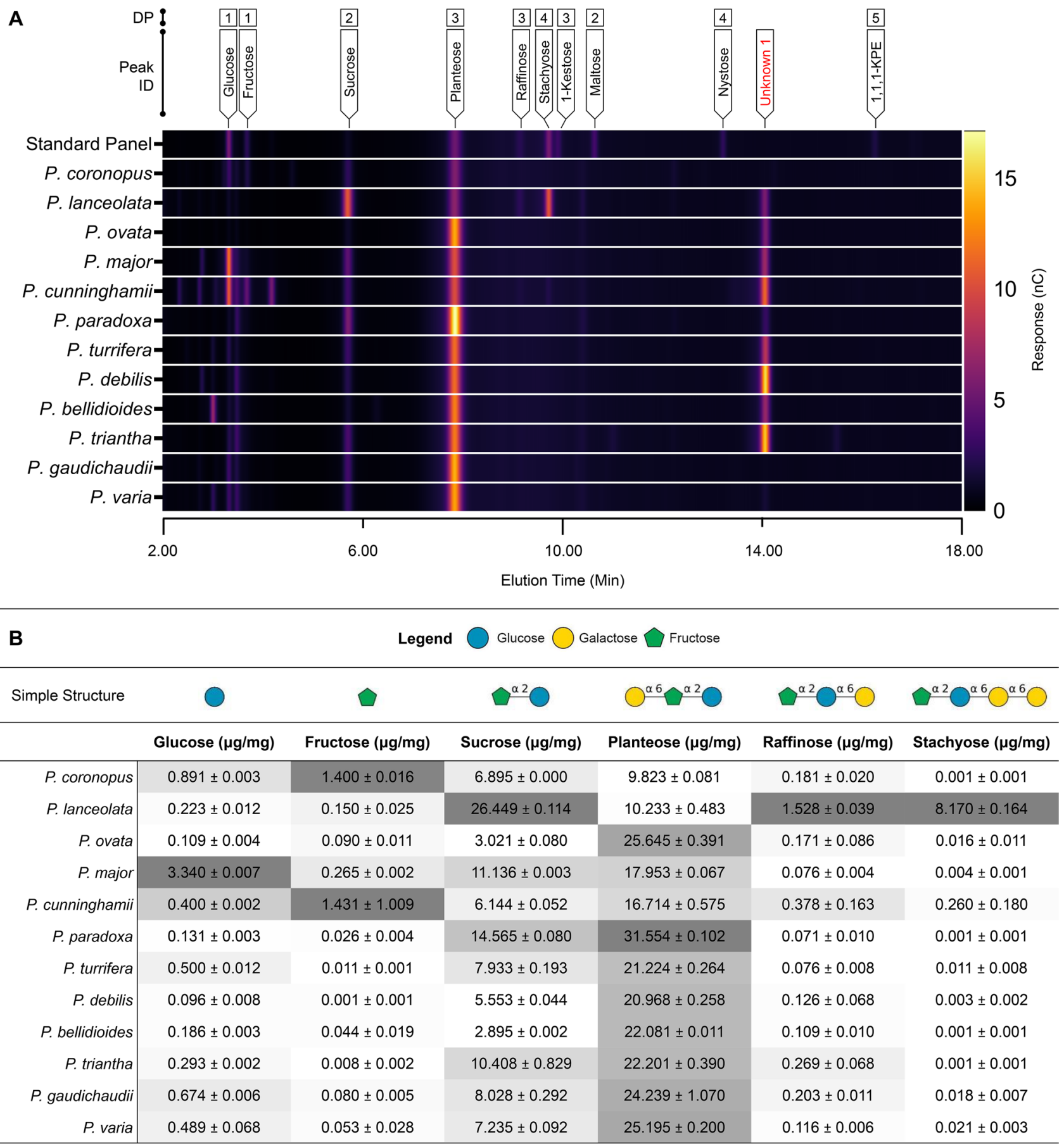

Figure 6. Soluble sugar profiling of Plantago seeds. (A) Chromatographic data presented in heatmap form to highlight differences in chromatographic profile between species. Chromatographic data has been cropped to an elution period that includes saccharides with a low degree-of-polymerisation (DP) (2-18 min). For simplicity, chromatographic data of standard runs (standard panel: a combined low DP saccharide suite, planteose and stachyose) were combined. (B) Quantification of key low DP saccharides and their schematic structure. A grey scale (dark grey, highest value; white, lowest value) has been used to display differences in abundance of one type between species. Values are means and standard deviation of three replicates. Abbreviations: 1,1,1$\mathrm{KPE}=1,1,1-$ Kestopentaose.

Currently unidentified, we also detected an additional sugar, Unknown 1 (Fig. 6A), eluting at approximately $14 \mathrm{~min}$ in extracts of all species. Based on the relative chromatographic peak area, Unknown 1 appears to be a major soluble sugar in seven of the species tested, comparable to planteose amounts in $P$. debilis or $P$. triantha (Supplementary Fig. 3). Levels of other oligosaccharides included in the standard panel, 1-kestose, maltose, nystose, and 1,1,1-kestopentaose were minute or not detected in the species studied here. 
Seed Morphometric Characteristics

Length $(\mathrm{mm})$

Width (mm)

1000 Grain Weight (mg)

Seed Nutrient Composition

Energy $(\mathrm{kJ} / 100 \mathrm{~g})$

Protein (\%)

Carbohydrate (\%)

Dietary Fibre (\%)

Fat (\%)

Fatty Acid Composition of Seed Oil

Saturated

Myristic Acid (14:0) (\%)

Pentadecylic Acid (15:0) (\%)

Palmitic Acid (16:0) (\%)

Margaric Acid (17:0) (\%)

Stearic Acid (18:0) (\%)

Arachidic Acid (20:0) (\%)

Behenic Acid (22:0) (\%)

Lignoceric Acid (24:0) (\%)

Unsaturated

$\omega-3$

Alpha-Linolenic Acid (ALA) (18:3) (\%)

$\omega-6$

Linoleic Acid (18:2) (\%)

Eicosadienoic Acid (20:2) (\%)

$\omega-7$

Palmitoleic Acid (16:1) (\%)

Vaccenic Acid (18:1) (\%)

w-9

Oleic Acid (18:1) (\%)

Gondoic Acid (20:1) (\%)

$\omega-3: \omega-6$

Yield of ALA

(\% w/w of whole seed)

Seed Morphometric Characteristics

Length (mm)

Width (mm)

1000 Grain Weight (mg)

Seed Nutrient Composition

Energy (kJ/100 g)

Protein (\%)

Carbohydrate (\%)

Dietary Fibre (\%)

Fat (\%)

Fatty Acid Composition of Seed Oil

Saturated

Myristic Acid (14:0) (\%)

Pentadecylic Acid (15:0) (\%)

Palmitic Acid (16:0) (\%)

Margaric Acid (17:0) (\%)

Stearic Acid (18:0) (\%)

Arachidic Acid (20:0) (\%)

Behenic Acid (22:0) (\%)

Lignoceric Acid (24:0) (\%)

Unsaturated

Continued

Total (\%)

\begin{tabular}{|l|l|l|l|l|c|}
\hline $1.138 \pm 0.132$ & $2.679 \pm 0.164$ & $2.843 \pm 0.224$ & $1.144 \pm 0.060$ & $1.394 \pm 0.111$ & $1.556 \pm 0.095$ \\
\hline $0.740 \pm 0.075$ & $1.227 \pm 0.143$ & $1.448 \pm 0.108$ & $0.653 \pm 0.081$ & $0.822 \pm 0.044$ & $1.101 \pm 0.087$ \\
\hline $205 \pm 8$ & $1508 \pm 33$ & $1618 \pm 43$ & $172 \pm 5$ & $364 \pm 2$ & $411 \pm 17$ \\
\hline
\end{tabular}

\begin{tabular}{|l|l|r|}
\hline 1176.15 & 844.89 & 898.3 \\
\hline $19.91 \pm 2.34$ & $11.20 \pm 0.08$ & $11.42 \pm 1.63$ \\
\hline $1.92 \pm 0.02$ & $4.68 \pm 0.15$ & $2.91 \pm 0.11$ \\
\hline $31.4 \pm 2.99$ & $35.47 \pm 0.26$ & $51.41 \pm 0.63$ \\
\hline $14.45 \pm 0.47$ & $7.76 \pm 0.54$ & $7.08 \pm 0.58$
\end{tabular}

1064.17

1260.15

\begin{tabular}{|l|l}
$16.36 \pm 0.35$ & $19.53 \pm 0.20$
\end{tabular}

\begin{tabular}{l|l}
$3.28 \pm 0.01$ & $2.63 \pm 0.58$
\end{tabular}

\begin{tabular}{|l|l}
$34.19 \pm 1.13$ & $48.49 \pm 2.52$ \\
\hline
\end{tabular}

\begin{tabular}{|l|l}
$12.70 \pm 0.44$ & $13.14 \pm 1.07$
\end{tabular}

1239.47

$31.35 \pm 2.16$

$4.63 \pm 0.04$

\begin{tabular}{l|l|l}
$14.45 \pm 0.47$ & $7.76 \pm 0.54$ & $7.08 \pm 0.58$
\end{tabular}

$16.85 \pm 0.16$

$22.39+0.37$

$17.20 \pm 0.14$

\begin{tabular}{|l|l}
$14.37 \pm 1.33$ & $15.01 \pm 0.03$
\end{tabular}

$0.04 \pm 0.01$

$0.06 \pm 0.01$

$0.06 \pm 0.03$

$0.08 \pm 0.01$

$0.17 \pm 0.01$

$12.05 \pm 0.15$

$17.52 \pm 0.17$

$0.12 \pm 0.01$

$0.10 \pm 0.01$

$0.13 \pm 0.02$

$12.43 \pm 0.13$

$3.83 \pm 0.03$

$3.70 \pm 0.10$

$0.11 \pm 0.01$

$3.82 \pm 0.06$

$0.50 \pm 0.01$

$0.45 \pm 0.05$

$0.42 \pm 0.00$

$0.17 \pm 0.02$

$0.21 \pm 0.01$

$0.09 \pm 0.01$

$82.99 \pm 0.02$

$27.98 \pm 0.02$

$27.98 \pm 0.02$

$38.29 \pm 0.09$

$38.22 \pm 0.09$

$0.06 \pm 0.01$

$0.76 \pm 0.01$

$0.08 \pm 0.00$

$0.67 \pm 0.01$

$0.15 \pm 0.03$

$0.19 \pm 0.03$

$0.05 \pm 0.06$

$77.53 \pm 0.48$

$82.76 \pm 0.08$

$6.74 \pm 0.22$

$6.74 \pm 0.22$

$43.99 \pm 0.36$

$3.18 \pm 0.12$

$3.18 \pm 0.12$

$39.72 \pm 0.29$

$43.95 \pm 0.42$

$39.65 \pm 0.31$

$0.07 \pm 0.02$

$1.73 \pm 0.06$

$1.37 \pm 0.03$

$0.16 \pm 0.02$

$0.23 \pm 0.02$

$1.20 \pm 0.05$

$15.96 \pm 0.10$

$15.83 \pm 0.11$

$1.49 \pm 0.04$

$25.08 \pm 0.15$

$38.49 \pm 0.47$

$24.90 \pm 0.13$

$38.12 \pm 0.49$

$0.13 \pm 0.01$

$0.76 \pm 0.04$

$4.04 \pm 0.13$

$0.18 \pm 0.01$

$0.16 \pm 0.01$

P. turrifera

P. debilis

$0.36 \pm 0.02$

$0.11 \pm 0.04$

$0.23 \pm 0.01$

P. bellidioides

\begin{tabular}{l|l}
$0.05 \pm 0.02$ & $0.09 \pm 0.02$
\end{tabular}

$0.05 \pm 0.02$

$10.23 \pm 0.90$

$0.15 \pm 0.00$

$2.82 \pm 0.27$

$0.57 \pm 0.06$

$0.39 \pm 0.04$

$0.11 \pm 0.03$

$85.53 \pm 1.41$

$12.31 \pm 1.70$

$12.31 \pm 1.70$

$52.30 \pm 1.68$

$52.21 \pm 1.69$

$0.09 \pm 0.01$

$1.10 \pm 0.05$

$0.10 \pm 0.01$

$0.99 \pm 0.06$

$19.82 \pm 1.91$

$19.57 \pm 1.88$

$0.26 \pm 0.02$

$0.28 \pm 0.09$

$1.56 \pm 0.16$

P. triantha

$0.05 \pm 0.00$

$11.8 \pm 0.11$

$0.06 \pm 0.01$

$2.62 \pm 0.17$

$0.26 \pm 0.00$

$0.12 \pm 0.01$

$0.03 \pm 0.04$

$84.92 \pm 0.01$

$43.42 \pm 0.03$

$43.42 \pm 0.03$

$16.60 \pm 0.20$

$16.57 \pm 0.21$

$0.03 \pm 0.01$

$1.44 \pm 0.04$

$0.21 \pm 0.00$

$1.23 \pm 0.04$

$23.46 \pm 0.21$

$23.35 \pm 0.20$

$0.12 \pm 0.02$

$2.64 \pm 0.06$

$5.71 \pm 0.46$

$25.19 \pm 0.31$

$10.41 \pm 1.28$

\begin{tabular}{|l|l|}
\hline $1.562 \pm 0.044$ & $1.308 \pm 0.071$
\end{tabular}

$1.563 \pm 0.115$

$1.236 \pm 0.132$

P. gaudichaudi

$15.76 \pm 0.02$ $0.11 \pm 0.00$ $0.07 \pm 0.02$

$11.84 \pm 0.06$

$0.09 \pm 0.01$

$3.11 \pm 0.03$

$0.38 \pm 0.01$

$0.16 \pm 0.01$

$0.00 \pm 0.00$

$84.24 \pm 0.02$

$32.09 \pm 0.12$

$32.09 \pm 0.12$

$23.38 \pm 0.13$

$23.34 \pm 0.19$

$0.04 \pm 0.06$

$1.16 \pm 0.01$

$0.18 \pm 0.02$

$0.99 \pm 0.00$

$27.60 \pm 0.29$

$27.49 \pm 0.34$

$0.12 \pm 0.05$

$1.37 \pm 0.00$

$3.34 \pm 0.40$

$0.840 \pm 0.086$

$0.717 \pm 0.036$

$0.879 \pm 0.079$

$383 \pm 8$

\begin{tabular}{|l|l|}
\hline $1.236 \pm 0.132$ \\
\hline $0.818 \pm 0.07$ \\
\hline $274 \pm 4$
\end{tabular}

$2.820 \pm 0.292$

P. varia

$355 \pm 6$
$288 \pm 9$

\begin{tabular}{|l|l|}
\hline 1174.72 & 1109.7 \\
\hline $18.03 \pm 1.06$ & $13.80 \pm 0.07$ \\
\hline $2.98 \pm 0.10$ & $2.67 \pm 0.08$ \\
\hline $48.36 \pm 2.33$ & $42.39 \pm 0.35$ \\
\hline $11.51 \pm 0.81$ & $12.90 \pm 1.44$ \\
\hline
\end{tabular}

1162.47

$20.43 \pm 0.22$

1142.19

$2.53 \pm 0.0$

$40.80 \pm 0.10$

$12.07 \pm 0.04$

$30.92 \pm 0.35$

$30.92 \pm 0.35$

$35.38 \pm 0.84$

$7.48 \pm 1.78$

$1.490 \pm 0.134$

$1936 \pm 102$

$2.495 \pm 0.250$

$1.387 \pm 0.142$

$1761 \pm 24$

$11.51 \pm 0.81$

\begin{tabular}{|l|c|c|c|c|c|c|}
\hline Total (\%) & $14.37 \pm 0.67$ & $18.73 \pm 0.06$ & $15.71 \pm 0.11$ & $22.14 \pm 0.21$ & $13.44 \pm 0.45$ & $16.64 \pm 0.17$ \\
\hline & $0.10 \pm 0.02$ & $0.07 \pm 0.00$ & $0.09 \pm 0.00$ & $0.09 \pm 0.03$ & $0.11 \pm 0.03$ & $0.14 \pm 0.01$ \\
\hline & $0.05 \pm 0.01$ & $0.04 \pm 0.00$ & $0.06 \pm 0.01$ & $0.17 \pm 0.00$ & $0.05 \pm 0.00$ & $0.05 \pm 0.00$ \\
\hline & $11.59 \pm 0.45$ & $14.18 \pm 0.12$ & $12.64 \pm 0.08$ & $17.59 \pm 0.32$ & $9.99 \pm 0.32$ & $12.84 \pm 0.16$ \\
\hline & $0.09 \pm 0.02$ & $0.08 \pm 0.00$ & $0.08 \pm 0.01$ & $0.13 \pm 0.02$ & $0.11 \pm 0.01$ & $0.12 \pm 0.01$ \\
\hline & $2.07 \pm 0.18$ & $3.63 \pm 0.02$ & $2.18 \pm 0.08$ & $3.46 \pm 0.29$ & $2.62 \pm 0.13$ & $2.85 \pm 0.04$ \\
\hline & $0.24 \pm 0.00$ & $0.41 \pm 0.02$ & $0.30 \pm 0.02$ & $0.40 \pm 0.00$ & $0.35 \pm 0.01$ & $0.34 \pm 0.01$ \\
\hline & $0.12 \pm 0.01$ & $0.19 \pm 0.01$ & $0.22 \pm 0.03$ & $0.22 \pm 0.06$ & $0.13 \pm 0.00$ & $0.18 \pm 0.04$ \\
\hline & $0.10 \pm 0.00$ & $0.13 \pm 0.03$ & $0.15 \pm 0.03$ & $0.09 \pm 0.12$ & $0.09 \pm 0.02$ & $0.11 \pm 0.02$ \\
\hline & $85.58 \pm 0.59$ & $81.19 \pm 0.01$ & $84.24 \pm 0.04$ & $77.80 \pm 0.30$ & $86.43 \pm 0.33$ & $83.22 \pm 0.03$ \\
\hline
\end{tabular}

\begin{tabular}{|l|c|c|c|c|c|c|}
\hline Total (\%) & $14.37 \pm 0.67$ & $18.73 \pm 0.06$ & $15.71 \pm 0.11$ & $22.14 \pm 0.21$ & $13.44 \pm 0.45$ & $16.64 \pm 0.17$ \\
\hline & $0.10 \pm 0.02$ & $0.07 \pm 0.00$ & $0.09 \pm 0.00$ & $0.09 \pm 0.03$ & $0.11 \pm 0.03$ & $0.14 \pm 0.01$ \\
\hline & $0.05 \pm 0.01$ & $0.04 \pm 0.00$ & $0.06 \pm 0.01$ & $0.17 \pm 0.00$ & $0.05 \pm 0.00$ & $0.05 \pm 0.00$ \\
\hline & $11.59 \pm 0.45$ & $14.18 \pm 0.12$ & $12.64 \pm 0.08$ & $17.59 \pm 0.32$ & $9.99 \pm 0.32$ & $12.84 \pm 0.16$ \\
\hline & $0.09 \pm 0.02$ & $0.08 \pm 0.00$ & $0.08 \pm 0.01$ & $0.13 \pm 0.02$ & $0.11 \pm 0.01$ & $0.12 \pm 0.01$ \\
\hline & $2.07 \pm 0.18$ & $3.63 \pm 0.02$ & $2.18 \pm 0.08$ & $3.46 \pm 0.29$ & $2.62 \pm 0.13$ & $2.85 \pm 0.04$ \\
\hline & $0.24 \pm 0.00$ & $0.41 \pm 0.02$ & $0.30 \pm 0.02$ & $0.40 \pm 0.00$ & $0.35 \pm 0.01$ & $0.34 \pm 0.01$ \\
\hline & $0.12 \pm 0.01$ & $0.19 \pm 0.01$ & $0.22 \pm 0.03$ & $0.22 \pm 0.06$ & $0.13 \pm 0.00$ & $0.18 \pm 0.04$ \\
\hline & $0.10 \pm 0.00$ & $0.13 \pm 0.03$ & $0.15 \pm 0.03$ & $0.09 \pm 0.12$ & $0.09 \pm 0.02$ & $0.11 \pm 0.02$ \\
\hline & $85.58 \pm 0.59$ & $81.19 \pm 0.01$ & $84.24 \pm 0.04$ & $77.80 \pm 0.30$ & $86.43 \pm 0.33$ & $83.22 \pm 0.03$ \\
\hline
\end{tabular}




\begin{tabular}{|l|l|l|l|l|l|l|l|}
\hline \multicolumn{2}{|l|}{} & P. turrifera & P. debilis & P. bellidioides & P. triantha & P. gaudichaudii & P. varia \\
\hline $\boldsymbol{\omega}$-3 & Total (\%) & $54.46 \pm 0.71$ & $41.04 \pm 0.06$ & $41.35 \pm 0.19$ & $7.69 \pm 1.08$ & $46.23 \pm 0.29$ & $42.69 \pm 0.95$ \\
\hline Alpha-Linolenic Acid (ALA) (18:3) (\%) & & $54.46 \pm 0.71$ & $41.35 \pm 0.19$ & $41.35 \pm 0.19$ & $7.69 \pm 1.08$ & $46.23 \pm 0.29$ & $42.69 \pm 0.95$ \\
\hline $\boldsymbol{\omega}$-6 & Total (\%) & $11.30 \pm 0.16$ & $24.17 \pm 0.10$ & $18.67 \pm 0.01$ & $45.70 \pm 1.81$ & $15.93 \pm 0.14$ & $19.48 \pm 0.61$ \\
\hline Linoleic Acid (18:2) (\%) & $11.26 \pm 0.15$ & $18.63 \pm 0.01$ & $18.63 \pm 0.01$ & $45.65 \pm 1.74$ & $15.91 \pm 0.11$ & $19.44 \pm 0.60$ \\
\hline Eicosadienoic Acid (20:2) (\%) & $0.04 \pm 0.01$ & $0.04 \pm 0.00$ & $0.04 \pm 0.00$ & $0.05 \pm 0.07$ & $0.02 \pm 0.03$ & $0.04 \pm 0.01$ \\
\hline $\boldsymbol{\omega}$ & & $1.66 \pm 0.02$ & $1.03 \pm 0.00$ & $1.31 \pm 0.02$ & $1.73 \pm 0.03$ & $1.15 \pm 0.04$ & $1.14 \pm 0.03$ \\
\hline Palmitoleic Acid (16:1) (\%) & $0.26 \pm 0.02$ & $0.15 \pm 0.01$ & $0.15 \pm 0.01$ & $0.26 \pm 0.02$ & $0.15 \pm 0.01$ & $0.14 \pm 0.02$ \\
\hline Vaccenic Acid (18:1) (\%) & $1.40 \pm 0.01$ & $1.17 \pm 0.01$ & $1.17 \pm 0.01$ & $1.47 \pm 0.01$ & $1.00 \pm 0.02$ & $1.00 \pm 0.01$ \\
\hline $\boldsymbol{\omega}$-9 & $18.15 \pm 0.07$ & $14.96 \pm 0.15$ & $22.90 \pm 0.26$ & $22.68 \pm 3.17$ & $23.13 \pm 0.06$ & $19.91 \pm 0.34$ \\
\hline Oleic Acid (18:1) (\%) & $18.07 \pm 0.08$ & $22.74 \pm 0.25$ & $22.74 \pm 0.25$ & $22.52 \pm 3.13$ & $23.00 \pm 0.06$ & $19.77 \pm 0.33$ \\
\hline Gondoic Acid (20:1) (\%) & $0.08 \pm 0.01$ & $0.16 \pm 0.01$ & $0.16 \pm 0.01$ & $0.16 \pm 0.04$ & $0.13 \pm 0.01$ & $0.14 \pm 0.00$ \\
\hline $\boldsymbol{\omega}$-3: $\boldsymbol{\omega}$-6 & $4.85 \pm 0.09$ & $1.70 \pm 0.01$ & $2.24 \pm 0.04$ & $0.17 \pm 0.01$ & $2.90 \pm 0.01$ & $2.22 \pm 0.15$ \\
\hline Yield of ALA & Total (\%) & $6.27 \pm 0.52$ & $5.29 \pm 0.58$ & $4.99 \pm 0.00$ & $0.58 \pm 0.22$ & $4.07 \pm 0.42$ & $3.88 \pm 0.12$ \\
\hline
\end{tabular}

Table 1. Summary of morphometric and nutritional characteristics of seeds of 12 Plantago species. Values are means and standard deviation.

Morphometric and nutritional characteristics of Plantago seeds. Plantago seeds differ significantly in length, width and thousand grain weight (Table 1) and can be categorised as small- or large-seeded (Supplementary Fig. 4). Seed size is not origin-specific as both native and non-native species are included in the small- and large-seeded categories (Supplementary Fig. 4).

To determine if there is any nutritional value in Plantago seeds that is normally overlooked, the seed nutrient (protein, carbohydrate, dietary fibre, and fat) composition was determined (Table 1). There is a significant amount of interspecific variation in the content of each nutrient. Compared to commercial psyllium (P. ovata) which lies at the extremes in three of four nutrients (Supplementary Fig. 5), the other species studied have less dietary fibre but more fat and more protein, with native species tending to have the highest protein content. In carbohydrate content, $P$. ovata is close to the median. $P$. ovata and $P$. lanceolata have a lower energy content than all other species due to their low protein and fat content ( $900 \mathrm{~kJ}$ vs. $\sim 1100 \mathrm{~kJ})$.

After determining that Plantago seeds contain a modest fat content, we investigated the fatty acid composition of the seed oil (Table 1). The ratio of saturated (SFA) to unsaturated fatty acids (UFA) is relatively similar between species (Median =1:5.2) but six of eight native species were slightly higher in UFA content, up to $33 \%$ more than P. ovata in P. gaudichaudii (1:6.4). The saturated fatty acid profiles are very similar between species with palmitic (16:0) and stearic acid (18:0) accounting for at least 94\% of SFA species. The most variation in fatty acid profiles comes from differences in the UFA profile. Multivariate analysis shows that $89 \%$ of variance between the species is due to differences in the ratio of omega-3 to omega-6 UFAs (Supplementary Fig. 6). All native species except $P$. triantha separate from commercial psyllium and the naturalised species because they contain a much higher quantity of omega-3 UFA in the seed oil; a significant difference between the non-native and native groups ( $p=0.008$, Supplementary Table 3 ). Commercial psyllium seed contains the least omega-3 UFA $(3.2 \% \mathrm{w} / \mathrm{w}$ ) of the species studied followed by the naturalised species (at most $28 \% \mathrm{w} / \mathrm{w}$ in P. coronopus). This is in clear contrast to the native species which contain an average of $39 \%$ omega-3 UFA (w/w), up to $54.5 \%$ in $P$. turrifera. When calculated as the omega- 3 to omega- 6 ratio, all native species have ratios greater than 1 $(1.37-4.85)$ except $P$. trianth $a$ which has a very low ratio similar to non-native species which are all less than 1 (0.11-0.76). The yield of the major omega-3 UFA, alpha-linolenic acid (18:3) is particularly high in native species where, again, $P$. turrifera has the highest yield of $6.27 \%(\mathrm{w} / \mathrm{w})$. There is also a small amount of variation $(8 \%)$ from differences in the omega- 6 to omega- 9 UFA ratio. The omega- 6 to omega- 9 ratio was fairly similar between native species and much greater in naturalised species (up to 2.6 times greater than the native species average). Transaturated fatty acids were not present (data not shown).

\section{Discussion}

In this study we present a comprehensive profiling of major nutritional and functional components of mucilageproducing seeds of 12 Plantago species that grow in Australia.

Mucilage comprises a major part of Plantago seeds and is the main factor currently influencing its use as a food additive and digestive health ingredient. Natural variation in Plantago mucilage properties is apparent even in the expanded mucilage architecture (Fig. 2). When extracting the seed mucilage in sequential fractions there are significant differences in WAC, yield and resistance to extraction (Fig. 3) and such variation may be explained by compositional differences (Fig. 4). The most easily-extracted Plantago seed fraction (CWE) contains abundant pectic monosaccharides, rhamnose and galacturonic acid, which are minimal in subsequent fractions (Fig. 4C). This is in line with our previous findings which suggest that the pectin in highly hydrophilic first mucilage fractions acts to 'prime' and initiate mucilage expansion'. Subsequent mucilage fractions which are obtained using more intense extraction conditions contain mostly xylose and arabinose, constituents of heteroxylan, in varying molar ratios. Yu et al ${ }^{64}$ showed that the innermost layer(s) of mucilage in P. ovata was most gel-like as a result of high heteroxylan substitution complexity, corroborated in our previous publication ${ }^{9}$ and by others ${ }^{65,66}$. Here, we show the same trends in other Plantago species where the second and third (HWE and IAE) mucilage 
fractions (akin to the inner layers described by Yu et al. ${ }^{64}$ ) had the highest AX ratios, an indication of heteroxylan substitution complexity. Similarly, the HWE and IAE mucilage fractions of $P$. cunninghamii, $P$. turrifera, $P$. bellidioides, $P$. debilis, $P$. gaudichaudii and $P$. varia had particularly high AX ratios suggesting even more complex heteroxylan substitution (Fig. 3B), as indicated by minimal immunolabelling by LM11 because the unsubstituted/ lowly-substituted backbone epitope is not present (Fig. 2e, g, h, i, k, and l). The inner mucilage fractions in these species also represented a large proportion of the total mucilage (Fig. 3B) and analysis of the WAC of mucilage on intact seeds (Fig. 3C) found WAC to be strongly correlated with both HWE $(\mathrm{r}=0.62, p=0.032)$ and IAE content $(\mathrm{r}=0.69, p=0.013)$ but the strongest correlation was a combination of the two $(\mathrm{r}=0.73, p=0.006)$ (Fig. 3D). Furthermore, the mucilage of these species is still apparent as retained, swollen envelopes even after extraction (compared to P. lanceolata, for example) (Supplementary Fig. 7) showing that the robustness of seed mucilage in Australian Plantago species is likely due, at least in part, to heteroxylan structure and resultant intermolecular bonds. It should be noted however that mucilage characteristics and composition appear to be at least partly independent of phylogenetic lineage and non-native/native status $(p>0.05$, Supplementary Table 3). Instead, preliminary findings associating mucilage content and water absorption capacity with key environmental factors (Supplementary Fig. 8) suggest that these strong macromolecular properties may have developed to ensure germination success in climatically different Australian environments, as has already been demonstrated for Plantago $^{67}$ and other species ${ }^{68,69}$. Importantly, the strong macromolecular properties in some Australian Plantago species like $P$. turrifera or $P$. bellidioides may also give them improved functionality over commercial psyllium in food and health applications where strong rheological/viscous properties are advantageous.

Along with the seed mucilage, we have also shown here that the internal tissues of Plantago seeds contain a wide array of saccharide families that represent dietary fibres that are typically underutilised during commercial psyllium production. After a review of the literature, we found that while Plantago species are known to have a substantial endosperm ${ }^{70,71}$, its exact composition has not been defined. The hard endosperm cell walls of $P$. ovata were suggested to be comprised solely of reserve cellulose $\mathrm{f}^{72}$ but crystalline cellulose levels were found to be, at most, $4 \%(\mathrm{w} / \mathrm{w})^{73}$. A clue is found during P. ovata seed development where mannan synthases are highly expressed along with the accumulation of mannose monosaccharides ${ }^{74}$. Here we are the first to confirm that the endosperm of all Plantago species studied contains thickened cell walls that are rich in mannan (Fig. 5). The substantial endosperm cell walls, like other species $^{75}$, gives Plantago a 'hard-seeded' quality ${ }^{76,77}$ (for example, seeds of $P$. ovata require over four times the specific grinding energy compared to wheat ${ }^{42,78}$ ) but also results in a larger proportion of dietary fibre carbohydrates in the absence of $\operatorname{starch}^{73}$. In addition to the abundant endosperm polysaccharides, soluble sugars are also abundant in the Plantago seeds studied here (Fig. 6), in agreeance with previous reports ${ }^{79}$. Planteose (originally isolated from Plantago and named for the genus ${ }^{80}$ ) is the major soluble sugar in seeds of all Plantago species studied here except $P$. lanceolata where only sucrose is higher. While minute quantities of the oligosaccharides raffinose and stachyose were detected in all species, these sugars are only abundant in $P$. lanceolata seeds where stachyose levels were even similar to planteose. This agrees with previous findings that raffinose and stachyose were only detected in seeds of Plantago Sect. Arnoglossum of which $P$. lanceolata is the only member included here ${ }^{81}$. An additional sugar, Unknown 1 (Fig. 6A), was also found in all species. Based on relative chromatographic peak area, Unknown 1 appears to be a major soluble sugar in seven of the species tested, with levels even comparable to planteose in P. debilis or P. triantha (Supplementary Fig. 3). As the chromatographic method used here elutes soluble sugars roughly by their degree-of-polymerisation (DP), it is likely that Unknown 1 is a tetra- or pentasaccharide (DP4-5) as it elutes after nystose (DP4) but before $1,1,1$-kestopentaose (DP5). While the exact identity of this compound will be elucidated by mass spectrometry, we hypothesise that Unknown 1 is likely to be the tetrasaccharide sesamose $(\alpha$-D-galactopyranosyl- $(1 \rightarrow 6)-\alpha$-Dgalactopyranosyl-( $1 \rightarrow 6)-\beta$-D-fructofuranosyl- $(2 \rightarrow 1)-\alpha$-D-glucopyranoside), a higher DP homologue of planteose, which while not reported previously for Plantago, is found in tandem with planteose in other species ${ }^{82}$. The array of soluble sugars and mannan polysaccharides in internal tissues of Plantago seeds are likely to be the primary carbon source fuelling germination ${ }^{83,84}$, however they also have medicinal and nutritional significance as fermentable dietary fibres. Fermentable dietary fibres are consumed by beneficial bacteria in the human gut, producing short chain fatty acids like butyrate which is required to maintain colon health ${ }^{85}$ and there is also mounting evidence that modulating the microbiome through dietary fibre supplementation has significant effects on a wide range of disease indications (for review see Lynch \& Pedersen ${ }^{86}$ ). While $P$. ovata husk is not a fermentable dietary fibre and thus provides limited fermentation- and microbiome-related benefits ${ }^{87}$, whole $P$. ovata seeds are reported to be fermentable, increasing faecal butyrate content in a clinical trial ${ }^{88}$ and improving numerous digestibility markers compared to the husk in a pre-clinical study ${ }^{89}$. These findings demonstrate that at least one non-husk component in Plantago seeds is fermentable by gut microbiota. While its exact prebiotic properties are currently unknown ${ }^{56}$, planteose, along with its homologues and other soluble sugars in Plantago seeds are likely fermentation candidates as many oligosaccharides are readily fermentable by beneficial bacteria in the gastrointestinal tract ${ }^{90,91}$. Endosperm mannan is also possibly fermentable compared to husk as diverse mannans and mannooligosaccharides released from mannan digestion have been shown to be well-fermented by a human faecal inoculum ${ }^{92,93}$.

Within the cells of the endosperm are large aleurone grains and oil bodies which house the protein and fat content of the Plantago seeds. Protein levels are generally similar to previous reports of Plantago species (10-20\% $\mathrm{w} / \mathrm{w})^{42,94-96}$, but Australian Plantago species are generally higher compared to these reports and all are higher than commercial psyllium (P. ovata) (Table 1). P. paradoxa and P. triantha are very high in protein content, over $30 \%$ $(\mathrm{w} / \mathrm{w})$, which is higher than important grain legumes like chickpeas or lentil (24\% and $26.1 \%$, respectively $)^{97}$. Plantago protein has previously been reported to contain essential amino acids and is well digested ${ }^{95}$ showing the potential of Plantago seeds as a protein source. Seeds of Plantago species are also rich in fats and all Australian species contain more fat than commercial psyllium, $P$. ovata (Table 1). Of particular nutritional importance is the proportion of omega-3 and omega- 6 UFAs which mammals are unable to synthesise and are thus essential. 
There is a growing body of evidence that omega-3 fatty acids, particularly long-chain fatty acids (LCFAs) EPA and DHA commonly obtained from consuming fish, are protective against cardiovascular diseases ${ }^{98}$. However many researchers conclude that the world's ecosystems could not sustainably supply the population with enough fish to provide the recommended EPA and DHA intake ${ }^{99}$ and thus sufficient intake of plant-derived UFAs to allow endogenous LCFA production from these precursors is recommended as an alternative. As plant-derived omega-3 (anti-inflammatory) and omega-6 (pro-inflammatory) ${ }^{100,101}$ UFAs are competitively desaturated and elongated into LCFAs by the same pathways, it is important that the ratio of omega- 3 to omega- 6 is at the very least $1: 1^{102}$ to reduce the risk of inflammation. However an omega- 3 to omega- 6 intake ratio of $4: 1$ is suggested to be ideal for promoting heart, liver and gut health ${ }^{103}$. Here we show that Australian native Plantago seeds have omega- 3 to omega- 6 ratios greater than the minimum adequate ratio of 1:1 (Table 1 ) and after extensive review of the literature we report that $P$. turrifera has the highest ratio of omega- 3 to omega- 6 fatty acids reported for any seed (4.85:1). This is higher than benchmark species Linum usitatissimum (flax) or Salvia hispanica (chia), with 3.44:1 and 3.08:1, respectively ${ }^{104}$, although total fat content was $50 \%$ and $30 \%$ lower in P. turrifera than these species, respectively. Consumption of Plantago seeds, particularly those of Australian natives like P. turrifera, may therefore contribute to improving the undesirable omega- 3 to omega- 6 ratio that is common in modern, western diets, and thus diminish the associated health risks ${ }^{100,102}$.

\section{Conclusions and future directions}

In this study we have provided a more complete understanding of the composition and morphology of seeds of twelve Plantago species that grow in Australia and outline implications these differences may have for food and nutritional applications. We have shown that among the species studied here there is variation in seed mucilage content and macromolecular properties with some native species having a higher capacity to absorb water than commercial psyllium, a key predictor of polysaccharide properties. We hypothesise that these differences are a result of fraction- and species-specific differences in heteroxylan and pectin content/structure and may represent wide differences in hydrocolloid functionality that can be exploited in industry. By profiling the nutrient content of the inner Plantago seed tissues (endosperm and embryo), we show that current commercial production of psyllium in particular underutilises the nutritional value of whole Plantago seeds. The use of a whole seed Plantago product, particularly from those species with favourable mucilage properties, and that are native and adapted to harsh Australian conditions, may provide a more nutritious but still functional alternative to commercial psyllium husk. In this way, the use of a Plantago WSF could confer multiple benefits: (1) hydrophilic mucilage polysaccharides could rheologically modify food systems and improve their quality; (2) minimal fermentation of the mucilage polysaccharides could benefit metabolic and gastrointestinal disorders that are improved when the viscosity and consistency of the gut digesta is increased; and (3) fermentable fibres and lipids along with other phytonutrients derived from the internal seed tissues could contribute to the improvement of a wide range of microbiome-, inflammatory- and oxidative stress-related disorders.

Future work will investigate how the specific differences in composition and properties outlined here impact the functionality of Plantago WSFs in food technology and gut health and aim to unravel the drivers of natural variation. The differences in seed mucilage content and properties appear to be mostly independent of phylogenetic lineage, however preliminary data suggests that differences among Australian native Plantago species might have developed as an 'adaptive trait' to cope with water-deficit stress in arid Australian environments suggesting that climate might be an important factor. Future work will aim to validate these preliminary findings by scrutinising more Plantago accessions that encompass more of the genus' environmental range and provide insight into the intraspecific heterogeneity in the traits tested here. Probing this 'adaptive trait' hypothesis may aid in prospecting for ideally-adapted Plantago accessions with specific seed compositions for development as novel crops with tailored industrial end-uses.

\section{Data availability}

The datasets used and analysed during this work are available from the corresponding author upon reasonable request.

Received: 17 November 2020; Accepted: 26 May 2021

Published online: 16 June 2021

\section{References}

1. Samuelsen, A. B. The traditional uses, chemical constituents and biological activities of Plantago major L. A review. J. Ethnopharmacol. 71, 1-21 (2000).

2. Heimler, D., Isolani, L., Vignolini, P., Tombelli, S. \& Romani, A. Polyphenol content and antioxidative activity in some species of freshly consumed salads. J. Agric. Food Chem. 55, 1724-1729 (2007).

3. Çoban, T., Saltan, G., Sever, B. \& İşcan, M. Antioxidant activities of plants used in traditional medicine in Turkey. Pharm. Biol. 41, 608-613 (2008).

4. Zubair, M., Nybom, H., Lindholm, C., Brandner, J. M. \& Rumpunen, K. Promotion of wound healing by Plantago major L. leaf extracts-ex-vivo experiments confirm experiences from traditional medicine. Nat. Prod. Res. 30, 622-624 (2016).

5. Moreno-Salazar, S., Robles-Zepeda, R. \& Johnson, D. Plant folk medicines for gastrointestinal disorders among the main tribes of Sonora, Mexico. Fitoterapia 79, 132-141 (2008).

6. Gott, B. Plant Species used by Aborigines of South-Eastern Australia. (2006).

7. Low, T. Wild Food Plants of Australia. (Angus \& Robertson Publishers, 1988).

8. Maiden, J. A new indigenous food-plant. Agric. Gaz. NSW 9, 355 (1898).

9. Phan, J. et al. The novel features of Plantago ovata seed mucilage accumulation, storage and release. Sci. Rep. 10, 1-10 (2020).

10. Cowley, J. M. \& Burton, R. A. The goo-d stuff: Plantago as a myxospermous model with modern utility. New Phytol. 229, 1917-1923 (2021). 
11. Aghdaei, S., Aalami, M., Geefan, S. \& Ranjbar, A. Application of Isfarzeh seed (Plantago ovate L.) mucilage as a fat mimetic in mayonnaise. J. Food Sci. Technol. 51, 2748-2754 (2014).

12. Cappa, C., Lucisano, M. \& Mariotti, M. Influence of Psyllium, sugar beet fibre and water on gluten-free dough properties and bread quality. Carbohydr. Polym. 98, 1657-1666 (2013).

13. Haque, A. \& Morris, E. R. Combined use of ispaghula and HPMC to replace or augment gluten in breadmaking. Food Res. Int. 27, 379-393 (1994).

14. McRorie, J. W. et al. Psyllium is superior to docusate sodium for treatment of chronic constipation. Aliment. Pharmacol. Ther. 12, 491-497 (1998).

15. Anderson, J. W. et al. Cholesterol-lowering effects of psyllium intake adjunctive to diet therapy in men and women with hypercholesterolemia: Meta-analysis of 8 controlled trials. Am. J. Clin. Nutr. 71, 472-479 (2000).

16. Anderson, J. W., Allgood, L. D., Turner, J., Oeltgen, P. R. \& Daggy, B. P. Effects of psyllium on glucose and serum lipid responses in men with type 2 diabetes and hypercholesterolemia. Am. J. Clin. Nutr. 70, 466-473 (1999).

17. Bijkerk, C. J. et al. Soluble or insoluble fibre in irritable bowel syndrome in primary care? Randomised placebo controlled trial. BMJ 339, 613-615 (2009).

18. Han, N. et al. Optimization and antioxidant activity of polysaccharides from Plantago depressa. Int. J. Biol. Macromol. 93, 644-654 (2016).

19. Behbahani, B. A. et al. Plantago major seed mucilage: Optimization of extraction and some physicochemical and rheological aspects. Carbohydr. Polym. 155, 68-77 (2017).

20. Ye, C. L., Hu, W. L. \& Dai, D. H. Extraction of polysaccharides and the antioxidant activity from the seeds of Plantago asiatica L.. Int. J. Biol. Macromol. 49, 466-470 (2011).

21. Gong, L. et al. A novel alkali extractable polysaccharide from Plantago asiatic L. seeds and its radical-scavenging and bile acidbinding activities. J. Agric. Food Chem. 63, 569-577 (2015).

22. Harput, U. S., Genc, Y. \& Saracoglu, I. Cytotoxic and antioxidative activities of Plantago lagopus L. and characterization of its bioactive compounds. Food Chem. Toxicol. 50, 1554-1559 (2012).

23. Zhou, Q. et al. Identification and quantification of phytochemical composition and anti-inflammatory, cellular antioxidant, and radical scavenging activities of 12 Plantago species. J. Agric. Food Chem. 61, 6693-6702 (2013).

24. Huang, D. F. et al. Immunomodulatory activity of the seeds of Plantago asiatica L. J. Ethnopharmacol. 124, 493-498 (2009).

25. Huang, D., Nie, S., Jiang, L. \& Xie, M. A novel polysaccharide from the seeds of Plantago asiatica L. induces dendritic cells maturation through toll-like receptor 4. Int. Immunopharmacol. 18, 236-243 (2014).

26. Jiang, L. M., Nie, S. P., Zhou, H. L., Huang, D. F. \& Xie, M. Y. Carboxymethylation enhances the maturation-inducing activity in dendritic cells of polysaccharide from the seeds of Plantago asiatica L. Int. Immunopharmacol. 22, 324-331 (2014).

27. Yang, Q. et al. Plantago asiatica L. Seed extract improves lipid accumulation and hyperglycemia in high-fat diet-induced obese mice. Int. J. Mol. Sci. 18, 1-14 (2017).

28. Samout, N. et al. Beneficial effects of Plantago albicans on high-fat diet-induced obesity in rats. Biomed. Pharmacother. 84, $1768-1775$ (2016).

29. Galisteo, M. et al. A diet supplemented with husks of Plantago ovata reduces the development of endothelial dysfunction, hypertension, and obesity by affecting adiponectin and TNF-alpha in obese Zucker rats. J. Nutr. 135, 2399-2404 (2005).

30. Romero, A. L., West, K. L., Zern, T. \& Fernandez, M. L. The seeds from Plantago ovata lower plasma lipids by altering hepatic and bile acid metabolism in guinea pigs 1. Am. Soc. Nutr. Sci. 132, 1194-1198 (2002).

31. Govt India Dept of Commerce. Psyllium seed (isobgul) 12119013 Export : Commodity-wise (2017).

32. Govt India Dept of Commerce. Psyllium husk (isobgul) 12119032 Export : Commodity-wise (2017).

33. Phan, J. et al. Differences in glycosyltransferase family 61 accompany variation in seed coat mucilage composition in Plantago spp. J. Exp. Bot. 67, 6481-6495 (2016).

34. Jensen, J. K., Johnson, N. \& Wilkerson, C. G. Discovery of diversity in xylan biosynthetic genes by transcriptional profiling of a heteroxylan containing mucilaginous tissue. Front. Plant Sci. 4, 1-15 (2013).

35. Tucker, M. R. et al. Dissecting the genetic basis for seed coat mucilage heteroxylan biosynthesis in Plantago ovata using gamma irradiation and infrared spectroscopy. Front. Plant Sci. 8, 326 (2017).

36. Benaoun, F. et al. Structural characterization and rheological behavior of a heteroxylan extracted from Plantago notata Lagasca (Plantaginaceae) seeds. Carbohydr. Polym. 175, 96-104 (2017).

37. Saeedi, M., Morteza-Semnani, K. \& Sagheb-Doust, M. Evaluation of Plantago major L. seed mucilage as a rate controlling matrix for sustained release of propranolol hydrochloride. Acta Pharm. 63, 99-114 (2013).

38. Saeedi, M., Morteza-Semnani, K., Ansoroudi, F., Fallah, S. \& Amin, G. Evaluation of binding properties of Plantago psyllium seed mucilage. Acta Pharm. 60, 339-348 (2010).

39. Yin, J.-Y., Chen, H.-H., Lin, H.-X., Xie, M.-Y. \& Nie, S.-P. Structural features of alkaline extracted polysaccharide from the seeds of Plantago asiatica L. and its rheological properties. Molecules 21, 1-11 (2016).

40. Behbahani, B. A., Shahidi, F., Yazdi, F. T., Mortazavi, S. A. \& Mohebbi, M. Use of Plantago major seed mucilage as a novel edible coating incorporated with Anethum graveolens essential oil on shelf life extension of beef in refrigerated storage. Int. J. Biol. Macromol. 94, 515-526 (2017).

41. Addoun, N. et al. Structural features and rheological behavior of a water-soluble polysaccharide extracted from the seeds of Plantago ciliata Desf. Int. J. Biol. Macromol. 155, 1333-1341 (2020).

42. Ziemichód, A., Wójcik, M. \& Różyło, R. Seeds of Plantago psyllium and Plantago ovata: Mineral composition, grinding, and use for gluten-free bread as substitutes for hydrocolloids. J. Food Process Eng. 12931, 1-9 (2018).

43. Pejcz, E., Spychaj, R., Wojciechowicz-Budzisz, A. \& Gil, Z. The effect of Plantago seeds and husk on wheat dough and bread functional properties. LWT Food Sci. Technol. 96, 371-377 (2018).

44. Healey, A., Furtado, A., Cooper, T. \& Henry, R. J. Protocol: A simple method for extracting next-generation sequencing quality genomic DNA from recalcitrant plant species. Plant Methods 10, 1-8 (2014).

45. Sun, Y., Skinner, D. Z., Liang, G. H. \& Hulbert, S. H. Phylogenetic analysis of Sorghum and related taxa using internal transcribed spacers of nuclear ribosomal DNA. Theor. Appl. Genet. 89, 26-32 (1994).

46. Rønsted, N., Chase, M. W., Albach, D. C. \& Bello, M. A. Phylogenetic relationships within Plantago (Plantaginaceae): Evidence from nuclear ribosomal ITS and plastid trnL-F sequence data. Bot. J. Linn. Soc. 139, 323-338 (2002).

47. Tay, M. L., Meudt, H. M., Garnock-Jones, P. J. \& Ritchie, P. A. DNA sequences from three genomes reveal multiple long-distance dispersals and non-monophyly of sections in Australasian Plantago (Plantaginaceae). Aust. Syst. Bot. 23, 47-68 (2010)

48. Criscuolo, A. \& Gribaldo, S. BMGE (Block Mapping and Gathering with Entropy): A new software for selection of phylogenetic informative regions from multiple sequence alignments. BMC Evol. Biol. 10, 1-21 (2010).

49. Stamatakis, A. RAxML-VI-HPC: maximum likelihood-based phylogenetic analyses with thousands of taxa and mixed models Alexandros. Phylogenetics 22, 2688-2690 (2006).

50. McCartney, L., Marcus, S. E. \& Knox, J. P. Monoclonal antibodies to plant cell wall xylans and arabinoxylans. J. Histochem. Cytochem. 53, 543-546 (2005).

51. Cowley, J. M. et al. A small-scale fractionation pipeline for rapid analysis of seed mucilage characteristics. Plant Methods 16, $1-12(2020)$. 
52. Burton, R. A. et al. Over-expression of specific HvCslF cellulose synthase-like genes in transgenic barley increases the levels of cell wall $(1,3 ; 1,4)-\beta$-D-glucans and alters their fine structure. Plant Biotechnol. J. 9, 117-135 (2011).

53. Wood, J. et al. Genetic and environmental factors contribute to variation in cell wall composition in mature desi chickpea (Cicer arietinum L.) cotyledons. Plant Cell Environ. 41, 2195-2208 (2018).

54. Verspreet, J. et al. A simple and accurate method for determining wheat grain fructan content and average degree of polymerization. J. Agric. Food Chem. 60, 2102-2107 (2012).

55. Matros, A. et al. Genome-wide association study reveals the genetic complexity of fructan accumulation patterns in barley grain. J. Exp. Bot. 72, 2383-2402 (2021).

56. Xing, X. et al. Isolation and structural elucidation by 2D NMR of planteose, a major oligosaccharide in the mucilage of chia (Salvia hispanica L.) seeds. Carbohydr. Polym. 175, 231-240 (2017).

57. Cheng, K., Zhou, Y. \& Neelamegham, S. DrawGlycan-SNFG: A robust tool to render glycans and glycopeptides with fragmentation information. Glycobiology 27, 200-205 (2017).

58. Folch, J., Lees, M. \& Sloane Stanley, G. A simple method for the isolation and purification of total lipid from animal tissues. J. Biol. Chem. 226, 497-509 (1957).

59. Liu, G., Mühlhäusler, B. S. \& Gibson, R. A. A method for long term stabilisation of long chain polyunsaturated fatty acids in dried blood spots and its clinical application. Prostaglandins Leukot. Essent. Fat. Acids 91, 251-260 (2014).

60. Hammer, Ø., Harper, D. A. T. \& Ryan, P. D. PAST: Paleotological statistics software package for education and data analysis. Palaeontol. Electron. 4, 1-9 (2001).

61. Food and Agriculture Organisation of the United Nations. Calculation of the Energy Content of Foods - Energy Conversion Factors. (1998). http://www.fao.org/3/Y5022E/y5022e04.htm.

62. Marcus, S. E. et al. Restricted access of proteins to mannan polysaccharides in intact plant cell walls. Plant J. 64, 191-203 (2010).

63. Schultz, C. J. et al. Consumer and health-related traits of seed from selected commercial and breeding lines of industrial hemp, Cannabis sativa L. J. Agric. Food Res. 2, e100025 (2020).

64. Yu, L. et al. Multi-layer mucilage of Plantago ovata seeds: Rheological differences arise from variations in arabinoxylan side chains. Carbohydr. Polym. 165, 132-141 (2017).

65. Ren, Y., Yakubov, G. E., Linter, B. R., MacNaughtan, W. \& Foster, T. J. Temperature fractionation, physicochemical and rheological analysis of psyllium seed husk heteroxylan. Food Hydrocoll. 104, e105737 (2020).

66. Zhou, P. et al. Comparative study between cold and hot water extracted polysaccharides from Plantago ovata seed husk by using rheological methods. Food Hydrocoll. 101, 105465 (2020).

67. Teixeira, A., Iannetta, P., Binnie, K., Valentine, T. A. \& Toorop, P. Myxospermous seed-mucilage quantity correlates with environmental gradients indicative of water-deficit stress: Plantago species as a model. Plant Soil 446, 343-356 (2019).

68. Fabrissin, I., Cueff, G., Berger, A., Granier, F. \& Sallé, C. Natural variation reveals a key role for rhamnogalacturonan I in seed outer mucilage and underlying genes 1. Plant Physiol. 181, 1498-1518 (2019).

69. Bhatt, A., Santo, A. \& Gallacher, D. Seed mucilage effect on water uptake and germination in five species from the hyper-arid Arabian desert. J. Arid Environ. 128, 73-79 (2016).

70. Youngken, H. Textbook of pharmacognosy. (Blakiston, 1950).

71. Bruneton, J. Pharmacognosy, phytochemistry, medicinal plants. (Lavoisier, 1995).

72. Madgulkar, A., Rao, M. \& Warrier, D. Characterization of Psyllium (Plantago ovata) Polysaccharide and Its Uses. Polysaccharides 1, 1-17 (2014)

73. Phan, J. Xylans in Plantago species. University of Adelaide, Honours Thesis (University of Adelaide, 2012).

74. Jensen, J. K. et al. The DUF579 domain containing proteins IRX15 and IRX15-L affect xylan synthesis in Arabidopsis. Plant J. 66, 387-400 (2011).

75. Gong, X., Bassel, G. W., Wang, A. \& Greenwood, J. S. The emergence of embryos from hard seeds is related to the structure of the cell walls of the micropylar endosperm, and not to endo-b-mannanase activity. Ann. Bot. https://doi.org/10.1093/aob/mci269 (2005).

76. Primack, R. B. Reproductive effort in annual and perennial species of plantago (Plantaginaceae). Am. Nat. 114, 51-62 (1979).

77. Miao, S., Bazzaz, F. \& Primack, R. Persistence of maternal nutrient effects in Plantago major: The third generation. Ecology 72, 1634-1642 (1991).

78. Dziki, D. \& Laskowski, J. Influence of wheat grain mechanical properties on grinding energy requirements. TEKA Comm. Mot. Power Ind. Agric. 6, 45-52 (2006).

79. Ahmed, Z., Rizk, A. \& Hammoud, F. Phytochemical studies of Egyptian Plantago species. J. Pharm. Sci. 156, 1060-1062 (1965).

80. Wattiez, N. \& Hans, M. A holoside extracted from the seeds of Plantago major L. and Plantago ovata Forsk. Bull. Mem. Acad. R. Med. Belg. 8, 386-396 (1943).

81. Gorenflot, R. \& Bourdu, K. Critères biochimiques et taxonomie expérimentale du genre Plantago. Bull. la Société Bot. Fr. 109, 349-360 (1962).

82. Hatanaka, S. Oligosaccharides in the seeds of Sesamum indicum L. Arch. Biochem. Biophys. 82, 188-194 (1959).

83. Wakabayashi, T., Joseph, B., Yasumoto, S., Akashi, T. \& Aoki, T. Planteose as a storage carbohydrate required for early stage of germination of Orobanche minor and its metabolism as a possible target for selective control. J. Exp. Bot. 66, 3085-3097 (2015).

84. Jukes, C. \& Lewis, D. Planteose, the major soluble carbohydrate of seeds of Fraxinus excelsior. Phytochemistry 13, 1519-1521 (1974).

85. O'Grady, J., O'Connor, E. \& Shanahan, F. Review article: Dietary fibre in the era of microbiome science. Aliment. Pharmacol. Ther. 49, 506-515 (2019).

86. Lynch, S. \& Pedersen, M. The human intestinal microbiome in health and disease. N. Engl. J. Med. 375, 2369-2379 (2016).

87. McRorie, J. W. Psyllium is not fermented in the human gut. Neurogastroenterol. Motil. 27, 1681-1682 (2015).

88. Fernandez-Banares, F. et al. Randomized clinical trial of Plantago ovata seeds (dietary fiber) as compared with mesalamine in maintaining remission in ulcerative colitis. Am. J. Gastroenterol. 94, 427-433 (1999).

89. Leng-Peschlow, E. Plantago ovata seeds as dietary fibre supplement: Physiological and metabolic effects in rats. Br. J. Nutr. 66, 331-349 (1991).

90. Alander, M. et al. Effect of galacto-oligosaccharide supplementation on human faecal microflora and on survival and persistence of Bifidobacterium lactis Bb-12 in the gastrointestinal tract. Int. Dairy J. 11, 817-825 (2001).

91. Bruno-Barcena, J. \& Azcarate-Peril, M. Galacto-oligosaccharides and colorectal cancer: Feeding our intestinal probiome. J. Funct. Foods 12, 92-108 (2015).

92. Asano, I., Hamaguchi, K., Fujii, S. \& Iino, H. In vitro digestibility and fermentation of mannooligosaccharides from coffee mannan. Food Sci. Technol. Res. 9, 62-66 (2003).

93. Jonathan, M. C. et al. In vitro fermentation of 12 dietary fibres by faecal inoculum from pigs and humans. Food Chem. 133, 889-897 (2012).

94. Mohamed, I. K., Osama, M.A.-F., El-Salam, S. M. A. \& Mohamed, Z.E.-O. Biochemical studies on Plantago major L. and Cyamopsis tetragonoloba L. Int. J. Biodivers. Conserv. 3, 83-91 (2011).

95. Romero-Baranzini, A. L., Rodriguez, O. G., Yanez-Farias, G. A., Barron-Hoyos, J. M. \& Rayas-Duarte, P. Chemical, physicochemical, and nutritional evaluation of Plantago (Plantago ovata Forsk). Cereal Chem. 83, 358-362 (2006).

96. Davison, E. Seed utilization by harvester ants. In Ant-plant interactions 1-6 (1982). 
97. Iqbal, A., Khalil, I. A., Ateeq, N. \& Khan, M. S. Nutritional quality of important food legumes. Food Chem. 97, 331-335 (2006).

98. Tocher, D. R., Betancor, M. B., Sprague, M., Olsen, R. E. \& Napier, J. A. Omega-3 long-chain polyunsaturated fatty acids, EPA and DHA: Bridging the gap between supply and demand. Nutrients 11, 1-20 (2019).

99. Crawford, M. et al. Role of plant-derived omega-3 fatty acids in human nutrition. Ann. Nutr. Metab. 44, 263-265 (2000).

100. Harris, W. S. \& Harris, W. S. The Omega-6/Omega-3 ratio and cardiovascular disease risk: uses and abuses. Curr. Atheroscler. Rep. 8, 453-459 (2006).

101. Patterson, E., Wall, R., Fitzgerald, G. F., Ross, R. P. \& Stanton, C. Health implications of high dietary omega-6 polyunsaturated fatty acids. J. Nutr. Metab. 2012, 1-6 (2012).

102. Simopoulos, A. P. The importance of the ratio of omega-6 / omega-3 essential fatty acids. Biomed. Pharmacother. 56, 365-379 (2002).

103. Calder, P. C. n-3 Polyunsaturated fatty acids, inflammation, and inflammatory diseases. Am. J. Clin. Nutr. 83, 1505S-1519S (2006).

104. Sargi, S. C. et al. Antioxidant capacity and chemical composition in seeds rich in omega-3: chia, flax, and perilla. Food Sci. Technol. 33, 541-548 (2013).

\section{Acknowledgements}

The authors acknowledge Dr Jana Phan for her hard work that paved the way for this study and Dr Tina BiancoMiotto for continued support. The authors thank Kylie Neumann and Shi Fang (Sandy) Khor for excellent technical assistance, Dr Andrea Matros for valuable assistance with soluble sugar profiling, Dr Julian Schwerdt for advice on phylogenetic analysis, Sanjiv Satija and Mathieu Baes for assistance with protein analysis, and SAHMRI for lipid analysis. The authors appreciate the valuable conservation work of the partners of the Australian Seedbank Partnership, particularly the ANBG National Seed Bank, Tasmanian Seed Conservation Centre and Victorian Conservation Seedbank, the collectors and the curators who generously provided some of the seed samples to allow this project to happen.

\section{Author contributions}

J.M.C. and R.A.B. conceived the study. J.M.C. conducted the experiments and wrote the manuscript. L.A.O. performed thin section microscopy. R.A.B. contributed to data interpretation and writing the manuscript. All authors read, edited, and approved the final manuscript.

\section{Funding}

This work was supported the Australian Research Council Centres of Excellence in Plant Cell Walls (Grant No. 110001007) and Plant Energy Biology (Grant No. 140100008). JMC is supported by a PhD scholarship from the Australian Government's Research Training Program and more recently more recently by an ARC Linkage Grant (LP180100971).

\section{Competing interests}

The authors declare no competing interests.

\section{Additional information}

Supplementary Information The online version contains supplementary material available at https://doi.org/ 10.1038/s41598-021-92114-1.

Correspondence and requests for materials should be addressed to R.A.B.

Reprints and permissions information is available at www.nature.com/reprints.

Publisher's note Springer Nature remains neutral with regard to jurisdictional claims in published maps and institutional affiliations.

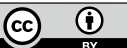

Open Access This article is licensed under a Creative Commons Attribution 4.0 International License, which permits use, sharing, adaptation, distribution and reproduction in any medium or format, as long as you give appropriate credit to the original author(s) and the source, provide a link to the Creative Commons licence, and indicate if changes were made. The images or other third party material in this article are included in the article's Creative Commons licence, unless indicated otherwise in a credit line to the material. If material is not included in the article's Creative Commons licence and your intended use is not permitted by statutory regulation or exceeds the permitted use, you will need to obtain permission directly from the copyright holder. To view a copy of this licence, visit http://creativecommons.org/licenses/by/4.0/.

(C) The Author(s) 2021 